\title{
On Dispersable Book Embeddings
}

\author{
Jawaherul Md. Alam ${ }^{1}$, Michael A. Bekos ${ }^{2}$, Martin Gronemann ${ }^{3}$, \\ Michael Kaufmann ${ }^{2}$, and Sergey Pupyrev ${ }^{1}$ \\ 1 Dep. of Computer Science, University of Arizona, Tucson, USA \\ $\{$ jawaherul, spupyrev\}@gmail.com \\ 2 Institut für Informatik, Universität Tübingen, Tübingen, Germany \\ \{bekos, mk\}@informatik. uni-tuebingen.de \\ 3 Institut für Informatik Universität zu Köln, Köln, Germany \\ gronemann@informatik. uni-koeln.de
}

\begin{abstract}
In a dispersable book embedding, the vertices of a given graph $G$ must be ordered along a line $\ell$, called spine, and the edges of $G$ must be drawn at different half-planes bounded by $\ell$, called pages of the book, such that: (i) no two edges of the same page cross, and (ii) the graphs induced by the edges of each page are 1-regular. The minimum number of pages needed by any dispersable book embedding of $G$ is referred to as the dispersable book thickness $\operatorname{dbt}(G)$ of $G$. Graph $G$ is called dispersable if $\operatorname{dbt}(G)=\Delta(G)$ holds (note that $\Delta(G) \leq d b t(G)$ always holds).

Back in 1979, Bernhart and Kainen conjectured that any $k$-regular bipartite graph $G$ is dispersable, i.e., $\operatorname{dbt}(G)=k$. In this paper, we disprove this conjecture for the cases $k=3$ (with a computer-aided proof), and $k=4$ (with a purely combinatorial proof). In particular, we show that the Gray graph, which is 3-regular and bipartite, has dispersable book thickness four, while the Folkman graph, which is 4-regular and bipartite, has dispersable book thickness five. On the positive side, we prove that 3-connected 3-regular bipartite planar graphs are dispersable, and conjecture that this property holds, even if 3-connectivity is relaxed.
\end{abstract}

\section{Introduction}

The book-embedding problem is a well studied problem in graph theory due to its numerous applications (see, e.g., $[9|17| 26 \mid 28$ ) with early results dating back in early 1970s 24. The input in this problem is a graph $G$ and the task is to find a linear order of the vertices of $G$ along a line $\ell$, called the spine of the book, and an assignment of the edges of $G$ to different half-planes, called pages of the book, delimited by the spine, such that no two edges of the same page cross (see Fig. 1a for an illustration). The minimum number of pages that are required by any book embedding of $G$ is commonly referred to as book thickness (but also as stack number or page number) and is denoted by $b t(G)$.

For planar input graphs, the literature is really rich. The most notable result is due to Yannakakis [30, who proved that the book thickness of a planar graph is at most four improving upon previous results 7[15. Better upper bounds are only known for restricted subclasses, such as planar 3-trees [15] (which 


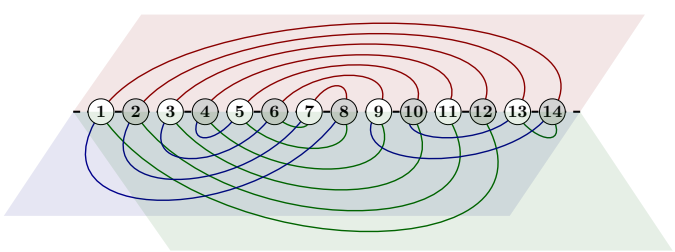

(a)

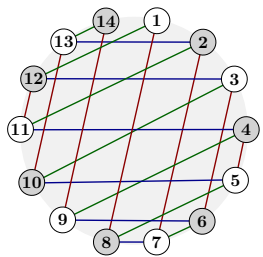

(b)

Fig. 1: (a) A dispersable book embedding of the 3-regular bipartite Heawood graph 31. with 3 pages (taken from [19]), and (b) an equivalent circular embedding with a 3-edgecoloring, in which no two edges of the same color cross.

fit in books with three pages), subgraphs of planar Hamiltonian graphs [4, 4connected planar graphs [23, planar graphs without separating triangles [20, Halin graphs [10], bipartite planar graphs [14, planar 2-trees 9], planar graphs of maximum degree 3 or 4 [1622] (which fit in books with two pages), and outerplanar graphs [4] (which fit in single-page books). Note that, in general, the problem of testing, whether a maximal planar graph has book thickness two, is equivalent to determining whether it is Hamiltonian, and thus is NP-complete [29].

For non-planar input graphs, the literature is significantly limited. It is known that the book thickness of a complete $n$-vertex graph is $\Theta(n)$ [4, while sublinear book thickness have all graphs with subquadratic number of edges 22, subquadratic genus [21] or sublinear treewidth [11. The book thickness is known to be bounded only for bounded genus graphs [21] and, more generally, all minorclosed graph families [5]. The reader is referred to [12] for a survey.

In this paper, we focus on dispersable book embeddings [4, in which the subgraphs induced by the edges of each page are additionally required to be 1-regular (i.e., matchings). The dispersable book thickness of a graph $G$, denoted by $\operatorname{dbt}(G)$, is defined analogously to the book thickness as the minimum number of pages required by any dispersable book embedding of $G$. So, by definition $\operatorname{dbt}(G) \geq \Delta(G)$ holds, where $\Delta(G)$ is the maximum degree of $G$. Finally, a graph $G$ is called dispersable if and only if $\operatorname{dbt}(G)=\Delta(G)$; see Fig. $1 \mathrm{a}$.

We note here that any book embedding with $k$ pages can be equivalently transformed into a circular embedding with a $k$-edge-coloring, in which no two edges of the same color cross, and vice versa 4[15. In the dispersable case, the graphs induced by the edges of the same color must additionally be 1-regular; see Fig. 1b. We refer to the order, in which the vertices appear along the boundary of a circular embedding with $\Delta(G)$ colors (or, equivalently along the spine of a dispersable book emdedding with $\Delta(G)$ pages), if any, as dispersable order.

Dispersable book embeddings were first studied by Bernhart and Kainen 4, who back in 1979 proved that the book thickness of the graph formed by the cartesian product of a dispersable bipartite graph $B$ and an arbitrary graph $H$ is upper bounded by the degree of $B$ plus the book thickness of $H$ (that is, $b t(B \times H) \leq b t(H)+\Delta(B)$ ), and posed the following conjecture (see also [19]): 
Conjecture 1 (Bernhart and Kainen, 1979). Every $k$-regular bipartite graph $G$ is dispersable, that is, $\operatorname{dbt}(G)=k$.

Clearly, Conjecture 1 holds for $k \leq 2$. As every $k$-regular bipartite graph admits a proper $k$-edge-coloring, Conjecture 1 implies that the dispersable book thickness of a regular bipartite graph equals its chromatic index. Overbay [25], who continued the study of dispersable embeddings in her Ph.D. thesis, observed that not every proper $k$-edge coloring yields a dispersable book embedding and that bipartiteness is a necessary condition in the conjecture of Bernhart and Kainen. She also proved that several classes of graphs are dispersable; among them trees, binary cube graphs, and complete graphs.

Our contribution: In Section 2, we disprove Conjecture 1 for the case $k=4$, by showing, with a purely combinatorial proof, that the Folkman graph (see Fig. 2), which is 4-regular and bipartite, has dispersable book thickness five. In Section 3, we first show how one can appropriately adjust a relatively recent SATformulation of the book embedding problem [3] for the dispersable case, and, using this formulation, we demonstrate that the Gray graph (see Fig. 13), which is 3-regular and bipartite, has dispersable book thickness four (thus, disproving Conjecture 1 also for the case $k=3$ ). Note that, since both graphs are not planar, their book thickness is at least three. Figs. 16a and 17 demonstrate that it is exactly three. In Section 4, we show that 3-connected 3-regular bipartite planar graphs are dispersable. Our findings lead to a number of interesting research directions, which we list in Section 5 , where we also conjecture that all (i.e., not necessarily 3 -connected) 3 -regular planar bipartite graphs are dispersable.

\section{The Dispersable Book Thickness of the Folkman Graph}

In this section, we study the book thickness of the Folkman graph [13, which can be constructed in two steps starting from $K_{5}$ as follows. First, we replace every edge by a path of length two to obtain a bipartite graph (see Fig. 2b). Then, we add for every vertex of the original $K_{5}$ a copy with the same neighborhood (see Fig. 2c). The resulting graph is the Folkman graph, which is clearly 4-regular and bipartite. We refer to a vertex of the original $K_{5}$ and to its copy as twin vertices. The remaining vertices of the Folkman, i.e., the ones obtained from the paths, are referred to as connector vertices. We denote the five pairs of twin vertices by $A_{1}, A_{2}, B_{1}, B_{2}, C_{1}, C_{2}, D_{1}, D_{2}, E_{1}, E_{2}$, and the ten connector vertices by ab, ac, ad, ae, bc, bd, be, cd, ce, de; see Fig. 2c.

To prove that the dispersable book thickness of the Folkman graph is five, it suffices to prove that its dispersable book thickness cannot be four, and that it admits a dispersable book embedding with five pages. For the later, refer to Fig. 16b in Appendix A. For the former, we will assume for a contradiction that the Folkman graph admits a circular embedding with a 4-edge-coloring, in which (i) no two edges of the same color cross, and (ii) the graphs induced by the edges of the same color are 1-regular. Since by Property (ii) adjacent edges must have different colors, we name them "crossing" such that we can use Property (i) also 


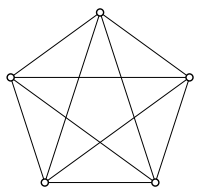

(a)

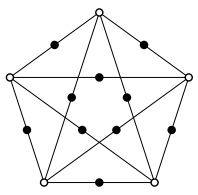

(b)

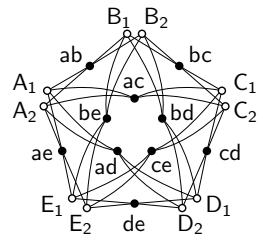

(c)

Fig. 2: Construction steps for the Folkman graph [13].

for them. In the drawings, we use red, green, blue, and orange to indicate the four colors of the edges; black is used for an unknown (or not yet specified) color; see, e.g., Fig. 3. For any subset of at least three twin or connector vertices of the Folkman graph, say $A_{1}$, ab and $B_{2}$, we denote the clockwise order in which they appear along the boundary of the circular embedding by $\left(\ldots A_{1} \ldots a b \ldots B_{2} \ldots\right)$. Every two vertices, say $a b$ and $A_{1}$, form two intervals, [ab, $\left.A_{1}\right]$ and $\left[A_{1}, a b\right]$, in the clockwise order that correspond to the two arcs on the circle.

Useful lemmas: In the following, we investigate properties of a dispersable book embedding with four pages of the Folkman graph. We start with a property that was first observed by Overbay [25] and later reproved by Hoske [18].

Lemma 1 (Overbay [25]). For any regular bipartite graph, the vertices from both partitions are alternating in a dispersable order.

For the Folkman graph, Lemma 1 implies that twin and connector vertices are alternating, i.e., for every pair of twins $A$ and $B$, interval $[A, B]$ contains a connector $x$, that is, the order is always $(\ldots A \ldots x \ldots B \ldots y \ldots)$. The next lemma strengthen the claim by describing an interval between twins $A_{1}$ and $A_{2}$.

Lemma 2. Let $\mathrm{A}_{1}, \mathrm{~A}_{2}$ be a pair of twins and $\left[\mathrm{A}_{1}, \mathrm{~A}_{2}\right],\left[\mathrm{A}_{2}, \mathrm{~A}_{1}\right]$ be two intervals defined by the twins. Then one of the following holds:

- one of the intervals contains exactly one connector vertex corresponding to the twins, and another one contains all other connectors, that is, the order is $\left(\ldots \mathrm{A}_{1}\right.$ ax $\mathrm{A}_{2}$... ay ... au ... av ...);

- both intervals contain two connectors corresponding to the twins (and possibly other connectors), that is, the order is (.. $\mathrm{A}_{1} \ldots \mathrm{ax} \ldots$ ay $\left.\ldots \mathrm{A}_{2} \ldots \mathrm{au} \ldots \mathrm{av} \ldots\right)$.

Proof. The twins $\mathrm{A}_{1}$ and $\mathrm{A}_{2}$ have four connectors, ax, ay, au, and av. Let us first show that it is impossible for one interval to contain all four connectors. Assume for a contradiction that interval $\left[A_{1}, A_{2}\right]$ contains all the connectors; the order is $\left(\ldots A_{1} \ldots\right.$ ax ...ay ...au ...av ... $\left.A_{2} \ldots\right)$. It is easy to see that five edges, $\left(A_{1}, a x\right)$, $\left(A_{1}, a y\right),\left(A_{1}, a u\right),\left(A_{1}, a v\right),\left(A_{2}, a x\right)$, are pairwise crossing. Thus, they need five distinct colors, which is impossible in a dispersable order of the Folkman graph.

To complete the proof, we find a contradiction for the case in which $\left[A_{1}, A_{2}\right]$ contains only one A's connector and some other connectors not adjacent to $A_{1}$ 


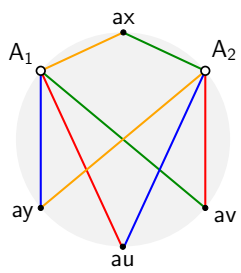

(a)

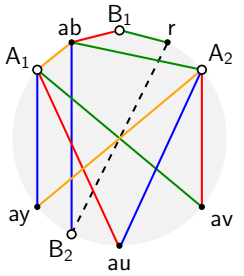

(b)

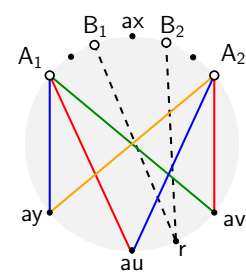

(c)

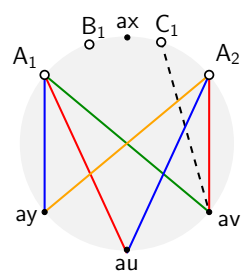

(d)

Fig. 3: Illustration for the proof of Lemma 2 .

and $A_{2}$. By Lemma 1. $\left[A_{1}, A_{2}\right]$ also contains other twin vertices. Denote $A$ 's connector in $\left[A_{1}, A_{2}\right]$ by ax and other three connectors by ay, au, av. Notice that the coloring of the eight A's edges is unique (up to color shift); see Fig. 3a. We distinguish the three cases depending on the twins lying in $\left[A_{1}, A_{2}\right]$ :

- Interval $\left[A_{1}, A_{2}\right]$ contains exactly one twin vertex, say $B_{1}$, that is, the order is $\left(\ldots A_{1} \cdot B_{1} \cdot A_{2} \ldots\right)$ with two intermediate connectors (by Lemma 1). It is easy to see that connector ab $\notin\left[A_{2}, A_{1}\right]$, as otherwise edge $\left(B_{1}, a b\right)$ cannot be colored. Hence, $a b \in\left[A_{1}, A_{2}\right]$; see Fig. $3 b$. Let $r$ be the second connector in $\left[A_{1}, A_{2}\right]$. Then, the green edge adjacent to $B_{1}$ must be $\left(B_{1}, r\right)$, and therefore edge $\left(B_{2}, r\right)$ cannot be colored; a contradiction.

- Interval $\left[A_{1}, A_{2}\right]$ contains exactly two same twins, say $B_{1}, B_{2}$, that is, the order is $\left(\ldots A_{1} \cdot B_{1} \cdot B_{2} \cdot A_{2} \ldots\right)$ with three intermediate connectors (by Lemma 1); see Fig. $3 \mathrm{c}$. If there is a connector $r$ of $B$ in the interval $\left[A_{2}, A_{1}\right]$, such that $r \neq a b$, then both edges $\left(B_{1}, r\right)$ and $\left(B_{2}, r\right)$ cannot be colored without forming five edges that pairwise cross, which is a contradiction. We claim that there is such connector. If $a b \in\left[A_{2}, A_{1}\right]$, then, since $a b \neq a x$, at most two connectors of $B$ can be on the interval $\left[A_{1}, A_{2}\right]$, which implies besides ab there is one more connector of $B$ in $\left[A_{2}, A_{1}\right]$, as desired. Otherwise, $a b \in\left[A_{1}, A_{2}\right]$. In this case, at most three connectors of $B$ can be on the interval $\left[A_{1}, A_{2}\right]$, which implies there is connector $r$ of $B$ in $\left[A_{2}, A_{1}\right]$, such that $r \neq a b$.

- Interval $\left[A_{1}, A_{2}\right]$ contains two or more different twins, say $B_{1}, C_{1}$, that is, the order is $\left(\ldots A_{1} \ldots B_{1} \ldots C_{1} \ldots A_{2} \ldots\right)$; see Fig. $3 \mathrm{~d}$. One of the connectors ab and ac is in $\left[A_{2}, A_{1}\right]$, since we assumed that $A_{1}$ and $A_{2}$ have only one connector on the opposite interval $\left[A_{1}, A_{2}\right]$. W.l.o.g. assume connector ac $\in\left[A_{2}, A_{1}\right]$. Then, the edge $\left(C_{1}, a c\right)$ cannot be colored; a contradiction.

Denote the number of A's connectors in $[x, y]$ by $\delta_{\mathrm{A}}(x, y)$. Lemma 2 defines two possible configurations for a pair of twins, $A_{1}$ and $A_{2}$. The first one, which we call 1-3 configuration, is when $\delta_{\mathrm{A}}\left(\mathrm{A}_{1}, \mathrm{~A}_{2}\right)=1$ and $\delta_{\mathrm{A}}\left(\mathrm{A}_{2}, \mathrm{~A}_{1}\right)=3$, that is, the first interval contains one connector and another interval contains three connectors. In that case, the twins have to lie next to each other in the order (that is, there is no other twins in between); we call such twins close. In the second configuration, called 2-2 configuration, $\delta_{\mathrm{A}}\left(\mathrm{A}_{1}, \mathrm{~A}_{2}\right)=\delta_{\mathrm{A}}\left(\mathrm{A}_{2}, \mathrm{~A}_{1}\right)=2$ holds. Here, the twins are called far (as there is at least one other twin in between). 


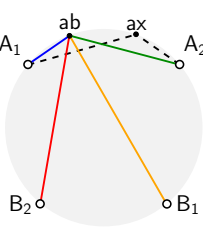

(a)

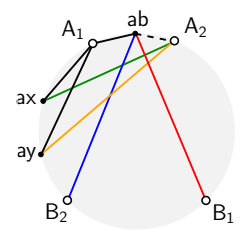

(b)

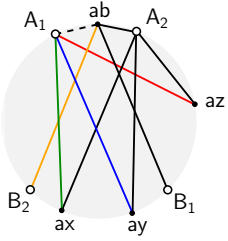

(c)

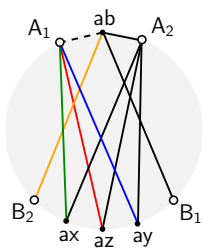

(d)

Fig. 4: Illustration for the proof of Lemma 3.

The next two lemmas describe properties of pairs of twins based on whether they alternate along the spine (crossing twin-pairs) or not (non-crossing twin-pairs).

Lemma 3 (non-crossing twin pairs). Let $\mathrm{A}_{1}, \mathrm{~A}_{2}$ and $\mathrm{B}_{1}, \mathrm{~B}_{2}$ be two pairs of non-crossing twins, that is, the order is $\left(\ldots \mathrm{A}_{1} \ldots \mathrm{A}_{2} \ldots \mathrm{B}_{1} \ldots \mathrm{B}_{2} \ldots\right)$. For connector ab, one of the following holds:

i. ab is in $\left[\mathrm{A}_{2}, \mathrm{~B}_{1}\right]$, that is, $\left(\ldots \mathrm{A}_{1} \ldots \mathrm{A}_{2} \ldots \mathrm{ab} \ldots \mathrm{B}_{1} \ldots \mathrm{B}_{2} \ldots\right)$;

ii. ab is in $\left[\mathrm{B}_{2}, \mathrm{~A}_{1}\right]$, that is, $\left(\ldots \mathrm{A}_{1} \ldots \mathrm{A}_{2} \ldots \mathrm{B}_{1} \ldots \mathrm{B}_{2} \ldots \mathrm{ab} \ldots\right)$;

iii. $\mathrm{A}_{1}$ and $\mathrm{A}_{2}$ are close twins and the four twins are separated by $\mathrm{A}$ 's connectors, that is, the order is $\left(\ldots \mathrm{A}_{1}\right.$ ab $\mathrm{A}_{2} \ldots \mathrm{ax} \ldots \mathrm{B}_{1} \ldots$ ay ... $\left.\mathrm{B}_{2} \ldots \mathrm{az} \ldots\right)$;

$i v . \mathrm{B}_{1}$ and $\mathrm{B}_{2}$ are close twins and the four twins are separated by $\mathrm{B}$ 's connectors, that is, the order is $\left(\ldots \mathrm{B}_{1}\right.$ ab $\mathrm{B}_{2} \ldots \mathrm{bx} \ldots \mathrm{A}_{1} \ldots$ by $\left.\ldots \mathrm{A}_{2} \ldots \mathrm{bz} \ldots\right)$.

Proof. If $a b$ is in $\left[A_{2}, B_{1}\right]$ or $\left[B_{2}, A_{1}\right]$, then the lemma holds. So, let ab be in $\left[A_{1}, A_{2}\right]$. If $A_{1}$ and $A_{2}$ are far, then by Lemma 2 there is another of $A$ 's connectors, say ax, in $\left[A_{1}, A_{2}\right]$. One of the edges $\left(A_{1}, a x\right),\left(A_{2}, a x\right)$ cannot be colored; see Fig. 4a. Thus, ab is the only of $A$ 's connector on $\left[A_{1}, A_{2}\right]$, and $A_{1}, A_{2}$ are close.

Twins $B_{1}$ and $B_{2}$ define three sub-intervals on $\left[A_{2}, A_{1}\right]$. If two of $A$ 's connectors, say ax and ay, belong to the leftmost sub-interval, then $\left(A_{2}, a x\right),\left(A_{2}\right.$, ay $)$, $\left(B_{1}, a b\right)$ and $\left(B_{2}, a b\right)$ pairwise cross, which implies that all must have different colors; see Fig. $4 \mathrm{~b}$. Thus, $\left(\mathrm{A}_{2}\right.$, ab) needs a fifth color; a contradiction. Symmetric is the case, in which two of A's connectors belong to the rightmost sub-interval. Finally, if two of A's connectors, say ax and ay, are on the central sub-interval, then by symmetry we may assume that the fourth of A's connectors, say az, belongs either to $\left[A_{2}, B_{1}\right]$ or to $\left[B_{1}, B_{2}\right]$. In both cases, edge $\left(B_{2}, a b\right)$ crosses $\left(A_{1}, a x\right)$, $\left(A_{1}\right.$, ay $)$ and $\left(A_{1}, a z\right)$, which implies that all must have different colors; see Figs. $4 \mathrm{c}$ and $4 \mathrm{~d}$. Thus, $\left(\mathrm{A}_{1}, \mathrm{ab}\right)$ needs a fifth color; a contradiction. We conclude that the three intervals contain one connector each, as in the claim.

Corollary 1. Let the order be $\left(\ldots \mathrm{A}_{1}\right.$ ab $\left.\mathrm{A}_{2} \ldots\right)$. Then $\mathrm{B}_{1}$ and $\mathrm{B}_{2}$ are far twins.

Proof. By Lemma 3 iii $B_{1}$ and $B_{2}$ are separated by $A$ 's connectors, that is, the order is $\left(\ldots A_{1}\right.$ ab $A_{2} \ldots$ ax $\ldots B_{1} \ldots$ ay $\ldots B_{2} \ldots$ az $\left.\ldots\right)$. If $B_{1}$ and $B_{2}$ are close, then the twins are in 1-3 configuration. Thus, the only connector between them is a B's connector, a contradiction. 


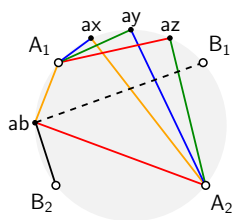

(a)

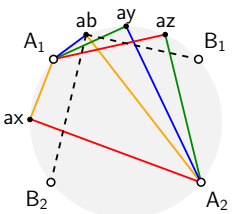

(b)

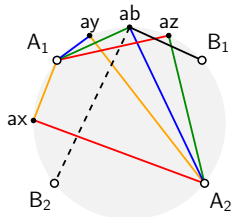

(c)

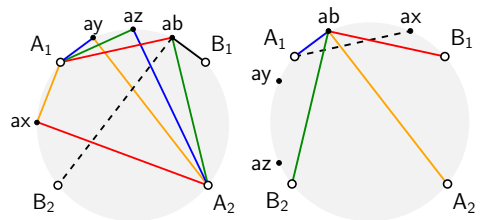

(d)

(e)

Fig. 5: Illustration for the proof of Lemma 4 .

Lemma 4 (crossing twin pairs). Let $\mathrm{A}_{1}, \mathrm{~A}_{2}$ and $\mathrm{B}_{1}, \mathrm{~B}_{2}$, be two pairs of crossing twins, that is, the order is $\left(\ldots \mathrm{A}_{1} \ldots \mathrm{B}_{1} \ldots \mathrm{A}_{2} \ldots \mathrm{B}_{2} \ldots\right)$. Then one of the following holds:

i. $\delta_{\mathrm{A}}\left(\mathrm{A}_{1}, \mathrm{~B}_{1}\right)=\delta_{\mathrm{A}}\left(\mathrm{B}_{1}, \mathrm{~A}_{2}\right)=\delta_{\mathrm{A}}\left(\mathrm{A}_{2}, \mathrm{~B}_{2}\right)=\delta_{\mathrm{A}}\left(\mathrm{B}_{2}, \mathrm{~A}_{1}\right)=1$;

ii. $\delta_{\mathrm{A}}\left(\mathrm{A}_{1}, \mathrm{~B}_{1}\right)=\delta_{\mathrm{A}}\left(\mathrm{A}_{2}, \mathrm{~B}_{2}\right)=2$ and $\delta_{\mathrm{A}}\left(\mathrm{B}_{1}, \mathrm{~A}_{2}\right)=\delta_{\mathrm{A}}\left(\mathrm{B}_{2}, \mathrm{~A}_{1}\right)=0$;

iii. $\delta_{\mathrm{A}}\left(\mathrm{A}_{1}, \mathrm{~B}_{1}\right)=\delta_{\mathrm{A}}\left(\mathrm{A}_{2}, \mathrm{~B}_{2}\right)=0$ and $\delta_{\mathrm{A}}\left(\mathrm{B}_{1}, \mathrm{~A}_{2}\right)=\delta_{\mathrm{A}}\left(\mathrm{B}_{2}, \mathrm{~A}_{1}\right)=2$.

In particular, $\mathrm{A}$ 's connectors are in the 2-2 configuration with respect to $\mathrm{B}$ 's twins, that is, $\delta_{\mathrm{A}}\left(\mathrm{B}_{1}, \mathrm{~B}_{2}\right)=\delta_{\mathrm{A}}\left(\mathrm{B}_{2}, \mathrm{~B}_{1}\right)=2$.

Proof. Besides the three cases described in the lemma, we will exclude all the remaining cases, which are as follows:

- There is an interval $I$ which is either $\left[\mathrm{A}_{1}, \mathrm{~B}_{1}\right]$, or $\left[\mathrm{B}_{1}, \mathrm{~A}_{2}\right]$ or $\left[\mathrm{A}_{2}, \mathrm{~B}_{2}\right]$ or $\left[\mathrm{B}_{2}, \mathrm{~A}_{1}\right]$ with $\delta_{\mathrm{A}}(I) \geq 3$,

- $\delta_{\mathrm{A}}\left(\mathrm{A}_{1}, \mathrm{~B}_{1}\right)=\delta_{\mathrm{A}}\left(\mathrm{B}_{2}, \mathrm{~A}_{1}\right)=2$ and $\delta_{\mathrm{A}}\left(\mathrm{B}_{1}, \mathrm{~A}_{2}\right)=\delta_{\mathrm{A}}\left(\mathrm{A}_{2}, \mathrm{~B}_{2}\right)=0$, and

- $\delta_{\mathrm{A}}\left(\mathrm{B}_{1}, \mathrm{~A}_{2}\right)=\delta_{\mathrm{A}}\left(\mathrm{A}_{2}, \mathrm{~B}_{2}\right)=2$ and $\delta_{\mathrm{A}}\left(\mathrm{A}_{1}, \mathrm{~B}_{1}\right)=\delta_{\mathrm{A}}\left(\mathrm{B}_{2}, \mathrm{~A}_{1}\right)=0$.

We start with the first case. Note that by Lemma $2, \delta_{\mathrm{A}}(I)$ cannot be 4 . Let w.l.o.g. $I=\left[\mathrm{A}_{1}, \mathrm{~B}_{1}\right]$ and assume for a contradiction that $\delta_{\mathrm{A}}(I)=3$. Then, by symmetry, we may assume $\delta_{A}\left(A_{2}, B_{2}\right)+\delta_{A}\left(B_{2}, A_{1}\right)=1$. It is easy to see that the three colors of the edges from $A_{2}$ to the three of the $A$ 's connectors in $\left[A_{1}, B_{1}\right]$, uniquely determine the four colors for the edges from $A_{1}$ to the A's connectors; see e.g., Fig. 5a. Then, we check the four possible locations for connector ab. For each case, we try to insert the two edges from ab to $B_{1}$ and $B_{2}$, and immediately achieve a contradiction; see Figs. $5 \mathrm{a} .5 \mathrm{~d}$ for an illustration.

For the second case, assume to the contrary that $\delta_{\mathrm{A}}\left(\mathrm{A}_{1}, \mathrm{~B}_{1}\right)=\delta_{\mathrm{A}}\left(\mathrm{B}_{2}, \mathrm{~A}_{1}\right)=2$ which directly implies that $\delta_{A}\left(B_{1}, A_{2}\right)=\delta_{A}\left(A_{2}, B_{2}\right)=0$. By symmetry, we may assume that connector $a b$ is in $\left[A_{1}, B_{1}\right]$, and that it appears before the second of $A$ 's connectors, say ax, in $\left[A_{1}, B_{1}\right]$, when moving along $\left[A_{1}, B_{1}\right]$ from $A_{1}$ to $B_{1}$; see Fig. 5 e. Since the edges from connector ab towards $A_{1}, A_{2}, B_{1}$ and $B_{2}$ use all colors, edge $\left(A_{1}, a x\right)$ cannot be colored; a contradiction. As the third case is symmetric to the second, the lemma follows.

Case analysis: We have now introduced the tools we need, and we proceed to we analyse several forbidden patterns, that is, subsequences of twins, that cannot occur in a dispersable order of the Folkman graph. 


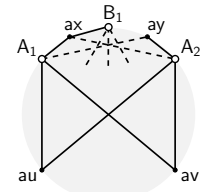

(a)

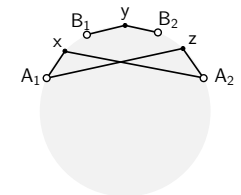

(b)

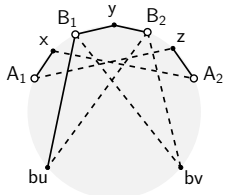

(c)

Fig. 6: Illustration for the proof of (a) Forbidden Pattern 1, (b)-(c) Forbidden Pattern2

Forbidden Pattern $1\left(\ldots \mathrm{A}_{1} \cdot \mathrm{B}_{1} \cdot \mathrm{A}_{2} \ldots\right)$ Between any twin pair, there is not exactly one single twin vertex.

Proof. Assume for a contradiction that there exists a pair of twins, $\mathrm{A}_{1}, \mathrm{~A}_{2}$, with exactly one twin vertex, $B_{1}$, between them in $\left[A_{1}, A_{2}\right]$. By Lemma 2 , there are two connectors of $A$ in $\left[A_{1}, A_{2}\right]$, call them ax and ay, and two connectors of $A$ in $\left[A_{2}, A_{1}\right]$, called them au and av; see Fig. 6a. Twin $B_{1}$ has four adjacent connectors, and only one of them can be a connector of $A$, namely ab. Hence, the three edges to the remaining connectors cross both $\left(A_{1}\right.$, ay $)$ and $\left(A_{2}\right.$, ax $)$; a contradiction.

Forbidden Pattern $2\left(\ldots \mathrm{A}_{1} \cdot \mathrm{B}_{1} \cdot \mathrm{B}_{2} \cdot \mathrm{A}_{2} \ldots\right)$ Between any twin pair, there are not exactly two same twin vertices.

Proof. Assume to the contrary that there exists a pair of twins, $A_{1}, A_{2}$, with exactly two same twin vertices, $B_{1}, B_{2}$, between them in $\left[A_{1}, A_{2}\right]$. By Lemma 1 . we assume that the order is $\left(\ldots A_{1} \times B_{1}\right.$ y $\left.B_{2} z A_{2} \ldots\right)$, where $x, y, z$ are connectors. By Lemma 2, two of them are connectors of $A$, including ab. If connector ab were $y$, then by Lemma 3 iv both $x$ and $z$ would have been connectors of $B$, contrading the fact that two of $x, y, z$ are connectors of $A$. Hence, ab is not $y$. It follows that there exist two $B$ 's connectors in $\left[A_{2}, A_{1}\right]$, call them bu and bv; see Fig. 6b. Now, it is easy to see that edges $\left(B_{1}, b v\right),\left(B_{2}, b u\right),(B, b v),\left(A_{1}, z\right)$, and $\left(A_{2}, x\right)$ pairwise cross; see Fig. 6c. So, they need five colors; a contradiction.

Forbidden Pattern $3\left(\ldots \mathrm{A}_{1} \cdot \mathrm{B}_{1} \cdot \mathrm{C}_{1} \cdot \mathrm{A}_{2} \ldots\right)$ Between any twin pair, there are not exactly two different twin vertices

Proof. Assume for a contradiction that there exists a pair of twins, $A_{1}, A_{2}$, with exactly two different twin vertices, $B_{1}, C_{1}$, between them in $\left[A_{1}, A_{2}\right]$. By Lemma 1 . we assume that the order is $\left(\ldots A_{1} \times B_{1}\right.$ y $\left.C_{1} z A_{2} \ldots\right)$, where $x, y, z$ are connectors. By Lemma 2, twins $A_{1}$ and $A_{2}$ have two connectors in $\left[A_{1}, A_{2}\right]$ and two connectors in $\left[A_{2}, A_{1}\right]$. By Lemma 3 applied first for twins $A$ and $D$, and then for $A$ and $E$, we conclude that ad and ae are on $\left[A_{2}, A_{1}\right]$, while $a b$ and ac are on $\left[A_{1}, A_{2}\right]$. By symmetry, we may assume that ae appears before ad on $\left[A_{2}, A_{1}\right]$.

Since A's and B's twins cross, by Lemma 4 we obtain that $\delta_{B}\left(A_{1}, A_{2}\right)=2$ and there exist two $B$ 's connectors in $\left[A_{1}, A_{2}\right]$. Symmetrically, there are two C's connectors in $\left[A_{1}, A_{2}\right]$. Hence, connector bc is in $\left[A_{1}, A_{2}\right]$. By symmetry, we consider two cases: either $b c=x \in\left[A_{1}, B_{1}\right]$ or $b c=y \in\left[B_{1}, C_{1}\right]$. 


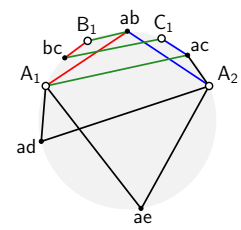

(a)

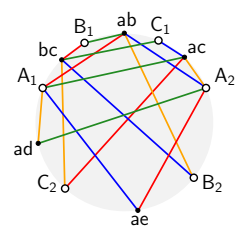

(b)

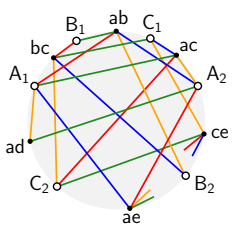

(c)

Fig. 7: Illustration of the case $b c=x \in\left[A_{1}, B_{1}\right]$.

- Assume first that $b c=x \in\left[A_{1}, B_{1}\right]$. Since there should exist an edge $\left(B_{1}, y\right)$ with the same color as $\left(C_{1}, b c\right)$, it follows that $y=a b$ and $z=a c$; see Fig. $7 a$, in which we have also assumed a coloring started w.l.o.g. with green $\left(A_{1}, a c\right)$, red $\left(A_{1}, a b\right)$, and blue $\left(A_{2}, a b\right)$. Since $\delta_{A}\left(B_{1}, A_{2}\right)=2$, by Lemma 4 liii applied for $A$ and $B$, we obtain that $\delta_{A}\left(A_{2}, B_{2}\right)=0$. So, $B_{2} \in\left[A_{2}\right.$, ae $]$. Since $\delta_{A}\left(A_{1}, C_{1}\right)=$ $\delta_{A}\left(C_{1}, A_{2}\right)=1$, by Lemma 4 i applied for $A$ and $C$, we have that $C_{2} \in$ [ae, ad]. Next we color the edges; see Fig. $7 b$ Edge $\left(B_{2}, a b\right)$ is orange (the remaining color for $a b)$, and thus, $\left(C_{2}, b c\right)$ and $\left(A_{2}, a c\right)$ are orange (otherwise two orange edges cross), while $\left(B_{2}, b c\right)$ is blue and $\left(C_{2}, a c\right)$ is red. Hence, $\left(A_{2}, a d\right)$ is green, $\left(A_{1}, a e\right)$ is blue, $\left(A_{1}, a d\right)$ is orange, and $\left(A_{2}, a e\right)$ is red.

Since $D_{1}$ and $D_{2}$ must be connected to ad via blue and red edges, $D_{1}$ and $D_{2}$ are in [ae, $\left.A_{1}\right]$. The orange edge from $C_{1}$ must end at a connector $\mathrm{cw} \notin\{\mathrm{ac}, \mathrm{bc}\}$ in $\left[A_{2}, B_{2}\right]$, and cx must have a green edge to $C_{2}$. Since $c w$ cannot be connected to [ae, $A_{1}$ ], where $D_{1}$ and $D_{2}$ reside (as it would cross blue edge $\left(A_{1}\right.$, ae) and red edge $\left.\left(A_{2}, a e\right)\right), c w \neq c d$ holds. Thus $c w=c e \in\left[A_{2}, B_{2}\right]$.

Since both $E_{1}$ and $E_{2}$ have to be connected to ce via blue and red edges, $E_{1}$ and $E_{2}$ are in $\left[A_{2}\right.$, ae $]$; see Fig. $7 \mathrm{c}$. In particular, one of them is in $\left[A_{2}, B_{2}\right]$ (because it must be connected to ce with a blue edge), while the other one is in $\left[\mathrm{B}_{2}, \mathrm{ae}\right]$ (because it must be connected to ae with an orange edge). Since both $D_{1}$ and $D_{2}$ are in $\left[a e, A_{1}\right]$, we conclude that $E_{1}, B_{2}, E_{2}$ form Forbidden Pattern $1\left(\ldots E_{1} \cdot B_{2} \cdot E_{2} \ldots\right)$, which is not possible.

- Assume now that $b c=y \in\left[B_{1}, C_{1}\right]$. Note that $x \neq a c$, as otherwise $B_{1}$ needs to have two edges in $\left[a c, C_{1}\right.$ ] (one with the color of $\left(a c, A_{2}\right)$ and one with the color of $\left.\left(a c, C_{1}\right)\right)$, which is impossible; see Fig. 8a. Since $a b, a c \in\left[A_{1}, A_{2}\right]$, it follows that $x=a b$ and $z=a c$; see Fig. $8 b$ where we have also assumed a coloring started w.l.o.g. with green $\left(A_{1}, a b\right)$, red $\left(A_{1}, a c\right)$, and blue $\left(A_{2}, a b\right)$.

What is the placement of $B_{2}$ and $C_{2}$ ? Applying Lemma 4 i for $B$ and $A$ and then for $C$ and $A$, we conclude that both $B_{2}$ and $C_{2}$ are in [ae, ad]. Edge $\left(a b, B_{2}\right)$ is either orange or green; assume w.l.o.g. orange, and thus (bc, $B_{2}$ ) is green. If $B_{2}$ appears before $C_{2}$ in [ae, ad], then the orange edge $\left(a b, B_{2}\right)$ must cross the edge $\left(b c, C_{2}\right)$; see Fig. $8 \mathrm{c}$. Hence, $\left(b c, C_{2}\right)$ needs a fifth color; contradiction. So, in the following we will assume that the order in [ae, ad] is $\left(\ldots\right.$ ae $\ldots C_{2} \ldots B_{2} \ldots$ ad $\left.\ldots\right)$. This order also fixes the colors of the following edges: $\left(C_{2}, b c\right)$ is orange, $\left(C_{2}, a c\right)$ is green, $\left(A_{2}, a c\right)$ is orange, $\left(A_{2}, a d\right)$ is red, $\left(A_{1}, a e\right)$ is blue, $\left(A_{1}, a d\right)$ is orange, $\left(A_{2}, a e\right)$ is green; see Fig. $8 \mathrm{~d}$. 


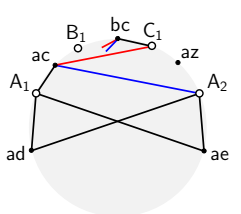

(a)

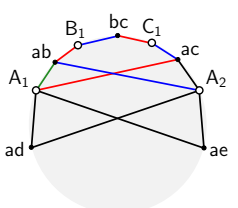

(b)

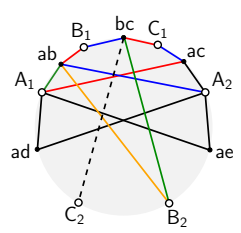

(c)

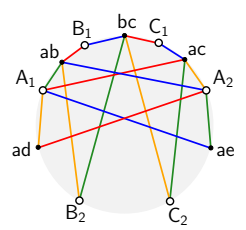

(d)

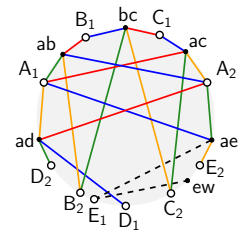

(e)

Fig. 8: Illustration of the case $b c=y \in\left[A_{1}, B_{1}\right]$.

Since bc $\in\left[\mathrm{B}_{1}, \mathrm{C}_{1}\right]$, by applying Lemma $3 \mathrm{i}$ twice, it follows that there exists a $B$ 's and a C's connector in $\left[C_{2}, B_{2}\right]$. Thus, by Lemma 1, there is also a twin vertex in $\left[C_{2}, B_{2}\right]$. Up to renaming, we may assume that this twin is $D_{1}$; observe that $D_{1}$ is connected to ad via blue edge. By Lemma 3 iii and iv applied for $D$ and $A$, we conclude that the second twin $D_{2}$ cannot be in [ad, $\left.A_{1}\right]$, because $B_{2} \in$ $\left[D_{1}, a d\right]$. Hence, by Lemma 3 i and ii $D_{2}$ is in $\left[A_{2}, a d\right]$. Since $D_{2}$ is connected to ad via green edge, we can further constraint the placement of $D_{2}$ in $\left[B_{2}, a d\right]$.

Since by Forbidden Pattern 1 the order $\left(\ldots D_{1} \cdot B_{2} \cdot D_{2} \ldots\right)$ is not possible, one of E's twins is in $\left[D_{1}, D_{2}\right]$, say w.l.o.g. $E_{1}$. By Lemma 3 liil and iv applied for $E$ and $A$, the second twin $E_{2}$ cannot be in $\left[A_{1}\right.$, ae], because $B_{2} \in\left[a e, E_{2}\right]$. Hence, $E_{2}$ is in [ae, $A_{1}$ ]. Now, observe that ae must be connected to either $E_{1}$ or $E_{2}$ with an orange edge. This connection cannot be with $E_{1}$, as othewrwise the orange edge (ae, $E_{1}$ ) would cross $\left(b c, C_{1}\right.$ ), which is also orange; a contradiction. It follows that ae is connected to $E_{2}$ with an orange edge, which further constraints the placement of $E_{2}$ to $\left[A_{2}, C_{2}\right]$; see Fig. $8 \mathrm{e}$. We assume that $E_{2}$ belongs to [ae, $\left.C_{2}\right]$; the case in which $E_{2}$ belongs to $\left[A_{2}, a e\right]$ is similar.

By Lemma 1. there is a connector in $\left[E_{2}, C_{2}\right]$. This connector cannot be a connector of $\mathrm{D}$, as otherwise both of its connections with $\mathrm{D}_{1}$ and $\mathrm{D}_{2}$ must be red; a contradiction. Similarly, we can argue that the connector in $\left[E_{2}, C_{2}\right]$ can neither be a connector of $\mathrm{B}$ nor a connector of $\mathrm{C}$. Thus, the connector in $\left[E_{2}, C_{2}\right]$ is a $E$ 's connector, call it ew. Since $E_{1}$ is connected to both ae and ew, the two edges $\left(E_{1}, a e\right)$ and $\left(E_{1}\right.$, ew $)$ need to cross a blue $\left(D_{1}\right.$, ad), a green $\left(\mathrm{C}_{2}, \mathrm{ac}\right)$, and an orange $\left(\mathrm{C}_{2}, \mathrm{bc}\right)$ edge, which is impossible.

Forbidden Pattern $4\left(\ldots \mathrm{A}_{1} \cdot \mathrm{B}_{1} \ldots \mathrm{B}_{2} \cdot \mathrm{A}_{2} \ldots\right)$ It is impossible to have a noncrossing pair of adjacent twins.

Proof. Assume to the contrary that $A_{1}, A_{2}$ and $B_{1}, B_{2}$ form a non-crossing pair of adjacent twins. Neither $A_{1}, A_{2}$ nor $B_{1}, B_{2}$ are close twins, as otherwise they would form Forbidden Pattern 2. Thus, by Lemma 3ii and ii $a b \in\left[A_{1}, B_{1}\right] \cup\left[B_{2}, A_{2}\right]$; say w.l.o.g. $a b \in\left[B_{2}, A_{2}\right]$. By Lemma 1 , there is a connector in $\left[A_{1}, B_{1}\right]$, which might be adjacent to one of $A_{1}$ or $B_{1}$ or not. We consider each case separately.

Assume first that the connector in $\left[A_{1}, B_{1}\right]$ is adjacent neither to $A_{1}$ nor to $B_{1}$; w.l.o.g. assume it is de. Since $A$ and $B$ are in 2-2 configuration, by Lemma 2 , there exists a $A$ 's connector, say ax, in $\left[B_{1}, B_{2}\right]$ and a $B$ 's connector, say by, 


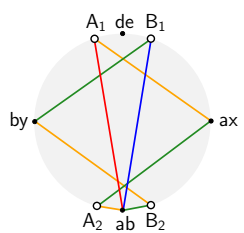

(a)

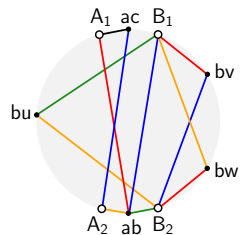

(b)

Fig. 9: Illustrations for the proof of Forbidden Pattern 4

in $\left[A_{2}, A_{1}\right]$; see Fig. 9a where we have also assumed a coloring started w.l.o.g. with red $\left(a b, A_{1}\right)$, orange $\left(a b, A_{2}\right)$, blue $\left(a b, B_{1}\right)$, and green $\left(a b, B_{2}\right)$. Then, edges $\left(A_{2}\right.$, ax $)$ and $\left(B_{1}\right.$, by $)$ have to be green, while $\left(B_{2}\right.$, by $)$ and $\left(A_{1}, a x\right)$ orange.

The two twins $X_{1}$ and $X_{2}$ adjacent to ax are in $\left[B_{1}, B_{2}\right]$, as they are connected to ax via red and blue edges. Similarly, twins $Y_{1}$ and $Y_{2}$ adjacent to by are in $\left[A_{2}, A_{1}\right]$. So, either $x$ is $d$ or e, or $y$ is $d$ or e. Further, we can conclude that the two connections from de to the interval $\left[B_{1}, B_{2}\right]$ go to the same twin pair, say $D_{1}, D_{2}$, while the two connections to the interval $\left[A_{2}, A_{1}\right]$ go to twin pair $E_{1}, E_{2}$.

If both $C_{1}$ and $C_{2}$ were either in $\left[A_{2}, A_{1}\right]$ or in $\left[B_{1}, B_{2}\right]$, then $E_{1}, E_{2}$ would form pattern $\left(\ldots A_{2} \cdot E_{1} \cdot E_{2} \cdot A_{1} \ldots\right)$, which is forbidden by Forbidden Pattern 2 . Hence, we may assume w.l.o.g. that $C_{1}$ is in $\left[A_{2}, A_{1}\right]$, while $C_{2}$ in $\left[B_{1}, B_{2}\right]$. Now, observe that at most one of the edges incident to $C_{1}$ might end in $\left[B_{1}, B_{2}\right]$, while its three remaining edges must end in $\left[A_{2}, A_{1}\right]$. A symmetric argument for $C_{2}$ implies that at most one of its incident edges might end in the opposite interval $\left[A_{2}, A_{1}\right]$, while its three remaining edges must end in $\left[B_{1}, B_{2}\right]$. Since twins $C_{1}$ and $\mathrm{C}_{2}$ share the same neighborhood, we have obtained a contradiction.

To complete the proof, we now consider the case in which the connector in $\left[A_{1}, B_{1}\right]$ is adjacent to one of $A_{1}$ or $B_{1}$; assume w.l.o.g. that it is ac (recall that $\left.a b \in\left[B_{2}, A_{2}\right]\right)$. Again, $A$ and $B$ are in 2-2 configuration. Since connector $a b$ is in $\left[B_{2}, B_{1}\right]$, by Lemma 2 , we may assume that $B$ has one additional connector, say bu, in $\left[B_{2}, B_{1}\right]$, and two connectors, say bv, bw, in $\left[B_{1}, B_{2}\right]$. Since ab $\in\left[B_{2}, A_{2}\right]$, ac $\in\left[A_{1}, B_{2}\right]$, and since $A_{1}, A_{2}$ and $B_{1}, B_{2}$ form a non-crossing pair of adjacent twins, bu $\in\left[A_{2}, A_{1}\right]$. Fig. $9 \mathrm{~b}$ illustrates the embedding with edge colors assigned as follows. W.l.o.g. $\left(a b, A_{1}\right)$ is red, $\left(a b, B_{1}\right)$ is blue, $\left(B_{2}, b u\right)$ is orange, and $\left(B_{1}\right.$, bu) is green. Then, $\left(A_{2}, a c\right)$ is blue, $\left(B_{2}, a b\right)$ is green, $\left(A_{2}, a b\right)$ is orange. It follows that $\left(B_{1}\right.$, bv $)$ and $\left(B_{2}\right.$, bw $)$ are red, $\left(B_{2}\right.$, bv $)$ is blue, and $\left(B_{1}\right.$, bw $)$ is orange.

Next, we consider connector bu and, as in the previous case, we observe that both twin vertices $U_{1}$ and $U_{2}$ that are adjacent to bu must be on the interval $\left[A_{2}, A_{1}\right]$. If there were no other twin vertex on the interval $\left[A_{2}, A_{1}\right]$, then $U_{1}$ and $\mathrm{U}_{2}$ would form pattern $\left(\ldots A_{2} \cdot U_{1} \cdot U_{2} \cdot A_{1} \ldots\right)$, which is forbidden by Forbidden Pattern 2. Assume w.l.o.g. that twin vertex $V_{1}$ is in $\left[A_{2}, A_{1}\right]$. We now claim that twin vertex $V_{2}$ is also in $\left[B_{1}, B_{2}\right]$. To see this, first observe that, by Lemma 1 . there exist at least one twin vertex in $\left[\mathrm{B}_{1}, \mathrm{~B}_{2}\right]$, since both connectors bv and bw belong to this interval. Now, assume for a contradiction that twin vertex 
$V_{2}$ is not in $\left[B_{1}, B_{2}\right]$. It follows that at most two twin vertices are in $\left[B_{1}, B_{2}\right]$, since $U_{1}, U_{2}, V_{1}, V_{2} \in\left[A_{2}, A_{1}\right]$. If there is only one twin vertex in $\left[B_{1}, B_{2}\right]$, say $W_{1}$, then pattern $\left(\ldots B_{1} \cdot W_{1} \cdot B_{2} \ldots\right)$ is formed, which is forbidden by Forbidden Pattern 1. If there are two twin vertices, say $W_{1}$ and $W_{2}$, in $\left[B_{1}, B_{2}\right]$, then pattern $\left(\ldots B_{1} \cdot W_{1} \cdot W_{2} \cdot B_{2} \ldots\right)$ is formed, which is forbidden by Forbidden Pattern 2 . Hence, twin vertex $V_{2}$ is in $\left[B_{1}, B_{2}\right]$, as claimed. Now, observe that out of the four edges incident to $V_{1}$ at least three have to end in the interval $\left[A_{2}, A_{1}\right]$, where $V_{1}$ resides. On the other hand, at most two edges incident to twin vertex $V_{2}$ may end in the opposite interval $\left[A_{2}, A_{1}\right]$. Since twins $V_{1}$ and $V_{2}$ share the same neighbourhood, we have obtained a contradiction. This concludes the proof.

Forbidden Pattern $5\left(\ldots \mathrm{A}_{1} \cdot \mathrm{B}_{1} \cdot \mathrm{C}_{1} \ldots \mathrm{A}_{2} \cdot \mathrm{B}_{2} \cdot \mathrm{C}_{2} \ldots\right)$ It is impossible to have $a$ crossing triple, i.e., a triple of consecutive twins that pairwise cross.

Proof. Assume to the contrary that there exists a crossing triple, and let the order be $\left(\ldots A_{1} \times B_{1}\right.$ y $C_{1} \ldots A_{2}$ u $\left.B_{2} \vee C_{2} \ldots\right)$, where $x, y, u, v$ are intermediate connectors. Observe that $A_{1}, A_{2}, B_{1}, B_{2}$, and $C_{1}, C_{2}$ form three pairs of crossing twins. By Lemma 4 , the number of $B$ 's connectors on opposite intervals formed by a pair of crossing twins is the same. Thus, the number of B's connectors in $\left[A_{1}, C_{1}\right]$ equals the number of B's connectors in $\left[A_{2}, C_{2}\right]$, which implies that in the union of the two intervals there are in total either four, or two, or zero B's connectors (i.e., four, two, or zero out of $x, y, u, v$ are B's connectors). We refer to the first and second case as non-zero crossing triple, while to the third as zero crossing triple. In Cases (i) and (ii) in the following we eliminate the case of non-zero crossing triples, and then assuming that there is no non-zero crossing triple we also eliminate the case of zero crossing triples.

i. B has four connectors among $\mathrm{x}, \mathrm{y}, \mathrm{u}, \mathrm{v}$. By symmetry, we may assume $\mathrm{x}=\mathrm{ab}$. This implies that there is not a $A$ 's connector in $\left[A_{2}, C_{2}\right]$. By Lemma 4 for $A$ and $C$, however, it follows that there must exist a $A$ 's connector in $\left[A_{2}, C_{2}\right]$; a contradiction.

ii. $\mathrm{B}$ has two connectors among $\mathrm{x}, \mathrm{y}, \mathrm{u}, \mathrm{v}$. By symmetry, we may assume that the B's connectors are $x \in\left[A_{1}, B_{1}\right]$ and $u \in\left[A_{2}, B_{2}\right]$. We will now prove by contradiction that $x \notin\{a b, b c\}$. Assume first that $x=b c$ and let w.l.o.g. the color of $\left(C_{1}, b c\right)$ be blue. Since $\left(C_{1}, b c\right)$ cannot be crossed by another blue edge, it follows that $\left(B_{1}, y\right)$ exists and is blue. This, however, contradicts the fact that $B$ has two connectors among $x, y, u, v$. Assume now that $x=a b$. Since $\delta_{A}\left(A_{1}, B_{1}\right)=1$, it follows by Lemma $4 \mathrm{i}$ that $\delta_{A}\left(A_{2}, B_{2}\right)=1$. Hence, $\mathrm{u} \in\left[\mathrm{A}_{2}, \mathrm{~B}_{2}\right]$ is a connector of $A$. Since $\mathrm{ab} \in\left[\mathrm{A}_{1}, \mathrm{~B}_{1}\right]$ and since by our initial assumption $\mathrm{u}$ is a connector of $\mathrm{B}$, we have again obtained a contradiction. It follows that either $\mathrm{x}=\mathrm{bd}$ or $\mathrm{x}=$ be holds. By symmetry, either $\mathrm{u}=\mathrm{bd}$ or $\mathrm{u}=$ be holds. By Lemma 2, there is a connector of $\mathrm{B}$ in each of $\left[\mathrm{C}_{1}, \mathrm{~A}_{2}\right]$ and $\left[C_{2}, A_{2}\right]$. W.l.o.g. assume ab is in $\left[C_{1}, A_{2}\right]$ and $b c$ is in $\left[C_{2}, A_{1}\right]$. It is easy now to see that the following edges pairwise cross: $\left(B_{1}, b c\right),\left(C_{1}, b c\right),\left(A_{1}, a b\right)$, $\left(\mathrm{B}_{1}, \mathrm{ab}\right)$, and $\left(\mathrm{u}, \mathrm{B}_{1}\right)$; a contradiction.

iii. B has zero connectors among $\mathrm{x}, \mathrm{y}, \mathrm{u}, \mathrm{v}$. By (i) and (ii), it follows that no nonzero crossing triple exists. By Lemma 4, two connectors of B exist in each 
of $\left[C_{1}, A_{2}\right]$ and $\left[C_{2}, A_{1}\right]$. Note that, $x$ is not a connector of $C$, as otherwise the four edges incident to $B_{1}$ would cross $\left(C_{1}, x\right)$. By symmetry, $u$ is not a connector of $C$, and $y$ and $v$ are not connectors of $A$. Also, ac $\notin\left[A_{1}, C_{1}\right] \cup$ $\left[\mathrm{A}_{2}, \mathrm{C}_{2}\right]$.

Let $\delta\left(C_{1}, A_{2}\right)$ and $\delta\left(C_{2}, A_{1}\right)$ be the number of twins in $\left[C_{1}, A_{2}\right]$ and $\left[C_{2}, A_{1}\right]$, respectively. Clearly, $\delta\left(C_{1}, A_{2}\right)+\delta\left(C_{2}, A_{1}\right) \leq 4$ holds. Since there exist two connectors of $B$ in each of $\left[C_{1}, A_{2}\right]$ and $\left[C_{2}, A_{1}\right]$, there exist at least one twin in each of $\left[C_{1}, A_{2}\right]$ and $\left[C_{2}, A_{1}\right]$. Thus, $\delta\left(C_{1}, A_{2}\right), \delta\left(C_{2}, A_{1}\right) \geq 1$. Assume w.l.o.g. that $D_{1} \in\left[C_{1}, A_{2}\right]$, and that $D_{1}$ encountered first in $\left[C_{1}, A_{2}\right]$.

The first twin encountered in $\left[C_{2}, A_{1}\right]$ cannot be $D_{2}$, as otherwise $B_{1}, C_{1}, D_{1}$, and $B_{2}, C_{2}, D_{2}$ would form a non-zero crossing triple containing connectors of $C$. By symmetry, let $E_{1}$ be the first twin in $\left[C_{2}, A_{1}\right]$.

We claim that $\delta\left(\mathrm{C}_{1}, \mathrm{~A}_{2}\right), \delta\left(\mathrm{C}_{2}, \mathrm{~A}_{1}\right) \leq 2$. For a contradiction, let $\delta\left(\mathrm{C}_{1}, \mathrm{~A}_{2}\right)=3$ (the case $\delta\left(C_{2}, A_{1}\right)=3$ is symmetric). Then, $\left[C_{1}, A_{2}\right]$ contains $D_{1}, D_{2}, E_{2}$. If $D_{2}$ precedes $E_{2}$ in $\left[C_{1}, A_{2}\right]$, then $E_{1}, A_{1}, B_{1}$, and $E_{2}, A_{2}, B_{2}$ form a non-zero crossing triple containing connectors of $A$. Otherwise, $D_{2}$ follows $E_{2}$ and thus $D_{1}, E_{2}, D_{2}$ form Forbidden Pattern 1 . Hence, our claim holds.

Since $D_{1} \in\left[C_{1}, A_{2}\right], E_{1} \in\left[C_{2}, A_{1}\right]$ and $\delta\left(C_{1}, A_{2}\right) \leq 2$, it follows that either $D_{1}, E_{2} \in\left[C_{1}, A_{2}\right]$ or $D_{1}, D_{2} \in\left[C_{1}, A_{2}\right]$ holds. In the former case, $D$ and $E$ form Forbidden Pattern 4 . In the later case, $E_{1}, E_{2} \in\left[C_{2}, A_{1}\right]$, and the order is $\left(A_{1} \cdot B_{1} \cdot C_{1} \cdot D_{1} \cdot D_{2} \cdot A_{2} \cdot B_{2} \cdot C_{2} \cdot E_{1} \cdot E_{2} \cdot\right)$. Now, recall that ac $\notin\left[A_{1}, C_{1}\right] \cup\left[A_{2}, C_{2}\right]$. By Lemma 22 it follows that ac $\notin\left[D_{1}, D_{2}\right]$ and ac $\notin\left[E_{1}, E_{2}\right]$. Hence, ac belongs to one of $\left[C_{1}, D_{1}\right],\left[D_{2}, A_{2}\right],\left[C_{2}, E_{1}\right],\left[E_{2}, A_{1}\right]$. Assume the former; the remaining cases are similar. In this case, $\left(C_{1}, a c\right)$ is crossed by the four edges incident to $B_{1}$, which is not possible.

Forbidden Pattern $6\left(\ldots \mathrm{A}_{1} \cdot \mathrm{B}_{1} \ldots \mathrm{A}_{2} \cdot \mathrm{B}_{2} \ldots\right)$ It is impossible to have a crossing pair of adjacent twins.

Proof. Assume to the contrary that $\mathrm{A}_{1}, \mathrm{~A}_{2}$ and $\mathrm{B}_{1}, \mathrm{~B}_{2}$ is a crossing pair of adjacent twins. By Forbidden Patterns 1 and 3 there exist at least two twins in each of $\left[B_{1}, A_{2}\right]$ and $\left[B_{2}, A_{1}\right]$; call them $X, Y, U, V$, and assume that the order is $\left(\ldots X \cdot A_{1} \cdot B_{1} \cdot Y \ldots U \cdot A_{2} \cdot B_{2} \cdot V \ldots\right)$. By Forbidden Pattern 3 each of $X, Y$ and $\mathrm{U}, \mathrm{V}$ are different twins. Let w.l.o.g. $\mathrm{X}=\mathrm{D}_{1}, \mathrm{Y}=\mathrm{C}_{1}$. Since $\mathrm{U}, \mathrm{V}$ are different, one of them, say w.l.o.g. $U$, is not a twin of $E$. So, either $U=C_{2}$ or $U=D_{2}$ holds. If $U=D_{2}$, then the order is $\left(\ldots D_{1} \cdot A_{1} \cdot B_{1} \ldots D_{2} \cdot A_{2} \cdot B_{2} \ldots\right)$, which implies that $\mathrm{D}, \mathrm{A}, \mathrm{B}$ form Forbidden Pattern 5 . We conclude that $\mathrm{U}=\mathrm{C}_{2}$ holds; see Fig. 10a.

Since the remaining twins are $D_{2}, E_{1}$, and $E_{2}$, and since one of these is $V$, there exist either zero, or one, or two twins in $\left[C_{1}, C_{2}\right]$. One yields Forbidden Pattern 1, while two yields either Forbidden Pattern 2 or Forbidden Pattern 3. depending on whether the two twins are same or not, respectively. Hence, we may assume that $C_{1}$ and $C_{2}$ are close twins.

Since $C_{1}$ and $C_{2}$ are close twins, twins $D_{2}, E_{1}$, and $E_{2}$ are all in $\left[B_{2}, D_{1}\right]$. Hence, their relative order in $\left[B_{2}, D_{1}\right]$ is: $\left(E_{1} \cdot D_{2} \cdot E_{2}\right)$, or $\left(E_{2} \cdot D_{2} \cdot E_{1}\right)$, or $\left(E_{1} \cdot E_{2} \cdot D_{2}\right)$, or $\left(E_{2} \cdot E_{1} \cdot D_{2}\right)$, or $\left(D_{2} \cdot E_{1} \cdot E_{2}\right)$, or $\left(D_{2} \cdot E_{2} \cdot E_{1}\right)$. The first two yield Forbidden Pattern 1. The next two yield Forbidden Pattern 2. By symmetry of the last two 


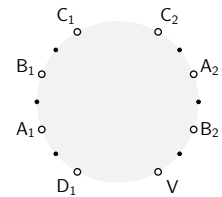

(a)

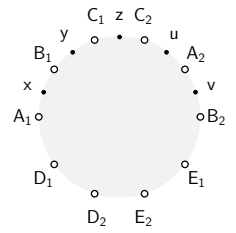

(b)

Fig. 10: Illustration for the proof of Forbidden Pattern 6

cases we may assume that the order is $\left(A_{1} \times B_{1}\right.$ y $\left.C_{1} z C_{2} u A_{2} \vee B_{2} \cdot E_{1} \cdot E_{2} \cdot D_{2} \cdot D_{1} \cdot\right)$, where $x, y, z, u, v$ are intermediate connectors; see Fig. $10 b$.

Since $C_{1}, C_{2}$ and $D_{1}, D_{2}$ are both close twins, by Corollary 1 , it follows that connector $z$, which is in $\left[C_{1}, C_{2}\right]$, is not cd. A symmetric argument on $C_{1}, C_{2}$ and $E_{1}, E_{2}$ implies that $z$ is not ce. By Lemma 2, $z$ is either ac or bc. By symmetry, we may assume $z=$ ac. Since ac $\in\left[C_{1}, C_{2}\right]$, by Lemma 3 iii and iv applied for $C$ and $A$, it follows that there exists a connector of $C$ at each of the intervals $\left[C_{1}, C_{2}\right],\left[C_{2}, A_{2}\right],\left[A_{2}, A_{1}\right]$ and $\left[A_{1}, C_{1}\right]$. Thus, connector $u \in\left[C_{2}, A_{2}\right]$ is a connector of C. Symmetrically, $y \in\left[B_{1}, C_{1}\right]$ is a connector of $C$. By Lemma 2 applied for $A$, there are two $A$ 's connectors in $\left[A_{1}, A_{2}\right]$; thus, $x \in\left[A_{1}, B_{1}\right]$ is a connector of $A^{4}$. Symmetrically, $v$ is a connector of $B$. Since $v \in\left[A_{2}, B_{2}\right]$ is a connector of $B$, by Lemma 4 it follows that $x \in\left[A_{1}, B_{1}\right]$ is also a connector of $B$, which implies that $x=a b$ (recall that $x$ is already shown to be a connector of $A$ ). Since $x \in\left[A_{1}, B_{1}\right]$ is a connector of $A$, again by Lemma 4 , it follows that $v \in\left[A_{2}, B_{2}\right]$ must be a connector of $A$. Since we have already shown that $v$ is a connector of $B$, it follows that $v=a b$. This is a contradiction, as $a b \in\left[A_{1}, B_{1}\right]$.

Forbidden Pattern $7\left(\ldots \mathrm{A}_{1} \cdot \mathrm{B}_{1} \cdot \mathrm{C}_{1} \cdot \mathrm{D}_{1} \cdot \mathrm{A}_{2} \ldots\right)$ Between any twin pair, it is impossible to have exactly three pairwise different twins.

Proof. Assume to the contrary that between $A_{1}$ and $A_{2}$ there exist exactly three pairwise different twins $B_{1}, C_{1}, D_{1}$, that is, the order is $\left(\ldots X \cdot A_{1} \cdot B_{1} \cdot C_{1} \cdot D_{1} \cdot A_{2}\right.$. $Y \ldots$ ), where $X$ and $Y$ are the twins preceding $A_{1}$ and following $A_{2}$. If $X=B_{2}$, then $A$ and $B$ form Forbidden Pattern 1 , if $X=C_{2}$, then $A$ and $C$ form Forbidden Pattern 3 if $X=D_{2}$, then $D$ and $A$ form Forbidden Pattern 6. Thus, $X=E_{1}$. By symmetry, $Y=E_{2}$ holds. But then $A$ and $E$ form Forbidden Pattern 4.

Forbidden Pattern $8\left(\ldots \mathrm{A}_{1} \cdot \mathrm{B}_{1} \cdot \mathrm{C}_{1} \cdot \mathrm{C}_{2} \cdot \mathrm{A}_{2} \ldots\right)$ Between any twin pair, it is impossible to have exactly three twins, such that two of them form a pair.

Proof. Assume to the contrary that between $A_{1}$ and $A_{2}$ there exist exactly three twins, such that two of them form a pair. Let $B_{1}, C_{1}, C_{2}$ be these twins. By

\footnotetext{
${ }^{4}$ Interval $\left[A_{1}, A_{2}\right]$ is the union of $\left[A_{1}, B_{1}\right],\left[B_{1}, C_{1}\right],\left[C_{1}, C_{2}\right]$, and $\left[C_{2}, A_{2}\right]$. As in the last three intervals there exist connectors of $C$ including ac $\in\left[C_{1}, C_{2}\right]$, it follows that the only interval, where the second connector of $A$ can be, is $\left[A_{1}, B_{1}\right]$.
} 


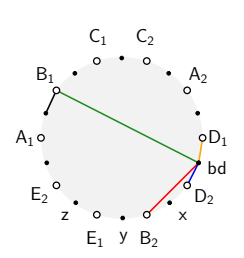

(a)

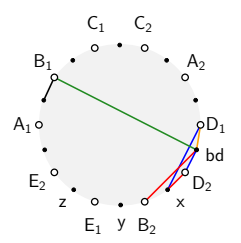

(b)

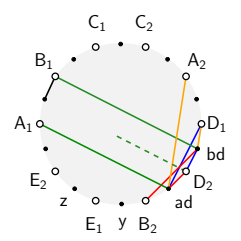

(c)

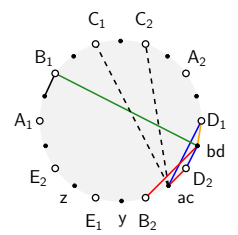

(d)

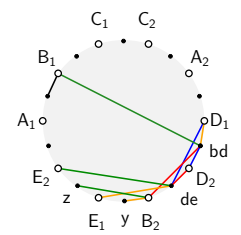

(e)

Fig. 11: Illustration for the proof of Forbidden Pattern 8

Forbidden Pattern 1. $C_{1}$ and $C_{2}$ are consecutive, that is, the order is $\left(A_{1} \cdot B_{1}\right.$. $\mathrm{C}_{1} \cdot \mathrm{C}_{2} \cdot \mathrm{A}_{2} \cdot \mathrm{U} \cdot \mathrm{V} \cdot \mathrm{X} \cdot \mathrm{Y} \cdot \mathrm{Z} \cdot$ ), where $\mathrm{U}, \mathrm{V}, \mathrm{X}, \mathrm{Y}$ and $\mathrm{Z}$ are the (remaining) twins following $A_{2}$. If $B_{2}=Z$, then $A$ and $B$ form Forbidden Pattern 1 if $B_{2}=Y$, then $A$ and $B$ form Forbidden Pattern 3 if $B_{2}=U$, then $A$ and $B$ form Forbidden Pattern 6. It follows that either $B_{2}=V$ or $B_{2}=X$ holds.

Assume first that $B_{2}=V$ holds. In this case, if twins $U$ and $X$ were of the same kind, then they would form Forbidden Pattern 11. Hence, $\mathrm{U}$ and $\mathrm{X}$ are different twins. Assume w.l.o.g. that $U=D_{1}$ and $X=E_{1}$. Thus, $\{Y, Z\}=\left\{D_{2}, E_{2}\right\}$. Clearly, if $D_{2}=Y$ and $E_{2}=Z$, then $D$ and $E$ form Forbidden Pattern 11. Thus, $E_{2}=Y$ and $D_{2}=Z$ holds. So, the order is $\left(A_{1} \cdot B_{1} \cdot C_{1} \cdot C_{2} \cdot A_{2} \cdot D_{1} \cdot B_{2} \cdot E_{1} \cdot E_{2} \cdot D_{2} \cdot\right)$. Now, it is not difficult to see that $A$ and $D$ form Forbidden Pattern 4.

To complete the proof, assume now that $B_{2}=X$. If twins $U$ and $V$ were different, say w.l.o.g. that $U=D_{1}$ and $V=E_{1}$, then $E_{1}$ and $E_{2}$ would form Forbidden Pattern 1 or 3 , as one of $Y$ and $Z$ must be $E_{2}$. Hence, $U$ and $V$ are same twins. Symmetrically, $Y$ and $Z$ are also same twins. Assume w.l.o.g. that $\mathrm{D}_{1}=\mathrm{U}, \mathrm{D}_{2}=\mathrm{V}, \mathrm{E}_{1}=\mathrm{Y}$, and $\mathrm{E}_{2}=\mathrm{Z}$, that is, the order is $\left(\mathrm{A}_{1} \cdot \mathrm{B}_{1} \cdot \mathrm{C}_{1} \cdot \mathrm{C}_{2} \cdot \mathrm{A}_{2}\right.$. $\left.D_{1} \cdot D_{2} \cdot B_{2} \cdot E_{1} \cdot E_{2} \cdot\right)$. Note that none of our forbidden patterns is violated.

By Lemma 2, the connector between $D_{1}$ and $D_{2}$ is a connector of $D$. Since $E$ and $C$ are close twins, it follows by Corollary 1 , that the connector between $D_{1}$ and $D_{2}$ is neither cd nor de. Hence, it is one of bd and ad; by symmetry, assume it is bd. Since $A$ and $B$ form a crossing twin pair and since bd $\in\left[A_{2}, B_{2}\right]$, by Lemma $41 \mathrm{i}$ it follows that $\delta_{B}\left(A_{1}, B_{1}\right)=1$, which implies that the connector in $\left[A_{1}, B_{2}\right]$ is a connector of $B$; see Fig. 11a, where we have assumed w.l.o.g. that $\left(B_{1}, b d\right)$ is green, $\left(B_{2}, b d\right)$ is red, $\left(D_{2}, b d\right)$ is blue, and $\left(D_{1}, b d\right)$ is orange.

Let $x, y, z$ be connectors in $\left[D_{2}, B_{2}\right],\left[B_{2}, E_{1}\right]$ and $\left[E_{1}, E_{2}\right]$. Since $\left(B_{2}, b d\right)$ cannot be crossed by another red edge, it follows that $\left(D_{2}, x\right)$ exists, and that it is red. Hence, $x$ is a connector of $D$, and it can be easily seen that $\left(D_{1}, x\right)$ is blue; Fig. $11 b$ for an illustration. Since $b d \in\left[D_{1}, D_{2}\right]$, connector $x$ is either ad, or $c d$, or de. If $x$ were ad, then the edge $\left(A_{2}, x\right)$ must be inevitably orange, which implies that edge $\left(A_{1}, x\right)$ is green. However, this color makes impossible to route a green edge for $D_{2}$, since the connector of $B$ in $\left[A_{1}, B_{1}\right]$ is not bd; see Fig. 11c. If $x$ were cd, then both edges $\left(C_{1}, x\right)$ and $\left(C_{2}, x\right)$ would have to cross the green edge $\left(B_{1}, d b\right)$. However, this is a contradiction again, since one of them must indeed be green and orange; see see Fig. $11 \mathrm{~d}$. Therefore, $x=$ de. This implies that edge $\left(B_{2}, y\right)$ 


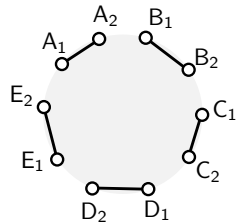

(a)

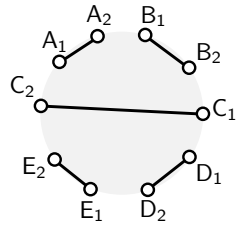

(b)

Fig. 12: Illustration for the proof of Theorem 1

exists and it is of the same color as $\left(E_{1}, d e\right)$. Similarly, edge $\left(B_{2}, z\right)$ exists and it is of the same color as $\left(E_{2}\right.$, de); see Fig. $11 \mathrm{e}$. Hence, both y and $z$ are connectors of $B$. So, including the connector of $B$ in the interval $\left[A_{1}, A_{2}\right]$ there exist in total three connectors of $B$ on the interval $\left[B_{2}, B_{1}\right]$, contradicting Lemma 2 .

Theorem 1. The dispersable book thickness of the Folkman graph is five.

Proof. To prove this theorem, it remains to conclude that the dispersable book thickness of the Folkman graph is not four. Let $d\left(\mathrm{~A}_{1}, \mathrm{~A}_{2}\right)$ be the number of twin vertices in $\left[\mathrm{A}_{1}, \mathrm{~A}_{2}\right]$, and let $d(\mathrm{~A})=\min \left(d\left(\mathrm{~A}_{1}, \mathrm{~A}_{2}\right), d\left(\mathrm{~A}_{2}, \mathrm{~A}_{1}\right)\right)$. By Forbidden Pattern 1, $d(\mathrm{~A}) \neq 3$; by Forbidden Patterns 2 and $3, d(\mathrm{~A}) \neq 4$; by Forbidden Patterns 7 and $8, d(\mathrm{~A}) \neq 5$. Therefore, either $d(\mathrm{~A})=2$ or $d(\mathrm{~A})=6$, that is, two twins are either close or are opposite in a dispersable order.

Assume now that there is a pair of twins, say $A_{1}, A_{2}$, that are opposite, and let $X, Y, Z, W$ be the twins in $\left[A_{1}, A_{2}\right]$. If twin $X$ and its counterpart were also opposite, then $A$ and $X$ would form Forbidden Pattern 4 . Hence, $X, Y$ are close. Symmetrically, Z, W are also close. Hence, at most one pair of twins are opposite.

Fig. 12 illustrates the remaining two cases, in which either no or one pair of twins are opposite. In the former case, by Lemma 2 there is a $A$ 's connector, say w.l.o.g. ab, in $\left[A_{1}, A_{2}\right]$. Then, by Corollary 1 , twins $B_{1}, B_{2}$ must be far; a contradiction.

To complete the proof, we find a contradiction for the case, in which there exists one pair of opposite twins, say w.l.o.g. $C_{1}, C_{2}$; see Fig. 12b. By Corollary 1 . the connector between each of the four close pairs can only be a connector of $C$. Hence, the order is $\left(C_{2} \times A_{1}\right.$ ac $A_{2}$ y $B_{1}$ bc $B_{2}$ z $C_{1}$ u $D_{1}$ dc $D_{2} \vee E_{1}$ ec $\left.E_{2} w\right)$, where $x, y, z, u, v, w$ are the remaining connectors in the order.

By Lemma 3 applied for $A$ and $C$, twins $C_{1}$ and $C_{2}$ are separated by $A$ 's connectors; that is, there exist $A$ 's connectors on both intervals $\left[A_{2}, C_{1}\right]$ and $\left[C_{2}, A_{1}\right]$. Thus, $x$ is a connector of $A$. Similarly, we conclude that $z$ is $B$ 's connector. Next observe that $z \neq a b$, otherwise five edges, $\left(a b, A_{1}\right),\left(a b, A_{2}\right),\left(a b, B_{1}\right),\left(b c, C_{2}\right)$, (bc, $C_{1}$ ), would pairwise cross. Hence, $y=a b$. Arguing symmetrically, we find that $\mathrm{v}=\mathrm{de}, \mathrm{u}$ is D's connector, and $\mathrm{w}$ is E's connector. That means that connector $b d$ is either $z$ or $u$. Both cases are impossible, as edges $\left(D_{1}, b d\right)$ or $\left(B_{2}, b d\right)$ would cross four $\mathrm{C}_{1}$ 's edges; a contradiction.

Corollary 2. The Folkman graph is not dispersable. 


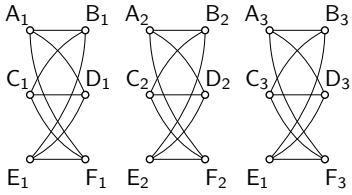

(a)

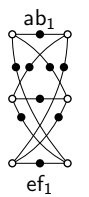

(b)

(b)

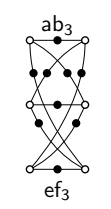

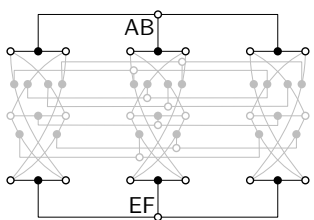

(c)

Fig. 13: Construction steps for the Gray graph [13: (a) the vertices of the three copies of $K_{3,3}$ are denoted by $\mathrm{A}_{i}, \mathrm{~B}_{i}, \mathrm{C}_{i}, \mathrm{D}_{i}, \mathrm{E}_{i}, \mathrm{~F}_{i} ; i=1,2,3$. (b) A vertex introduced in the first step between $X_{i}$ and $Y_{i}$ is denoted by $x y_{i}$. (c) A vertex introduced in the second step connecting $x y_{1}, x_{2}$ and $x y_{3}$ is denoted by $X Y$, where $X, Y \in\{A, B, C, D, F, E\}$.

\section{The Dispersable Book Thickness of the Gray Graph}

In this section, we study the book thickness of the Gray graph [6], which can be constructed in two steps starting from three copies of $K_{3,3}$ as follows. First, we subdivide every edge as we did also with the Folkman graph (see Fig. 13b). Then, for each newly introduced vertex $u$ in the first copy, with $v$ and $w$ being its counterparts in the other two copies, we add a new vertex connected to $u$, $v$ and $w$ (see Fig. 13c). The resulting graph is the Gray graph, which is clearly 3 -regular and bipartite.

Our computer-aided proof is based on appropriately adjusting a relatively recent formulation of the (ordinary) book embedding problem as a SAT instance [3. In this formulation, there exist three different types of variables, denoted by $\sigma, \phi$ and $\chi$, with the following meanings: (i) for a pair of vertices $u$ and $v$, variable $\sigma(u, v)$ is true, if and only if $u$ is to the left of $v$ along the spine, (ii) for an edge $e$ and a page $i$, variable $\phi_{i}(e)$ is true, if and only if edge $e$ is assigned to page $i$ of the book, and (iii) for a pair of edges $e$ and $e^{\prime}$, variable $\chi\left(e, e^{\prime}\right)$ is true, if and only if $e$ and $e^{\prime}$ are assigned to the same page. Hence, there exist in total $O\left(n^{2}+m^{2}+p m\right)$ variables, where $n$ denotes the number of vertices of the graph, $m$ its number of edges, and $p$ the number of available pages. An additional $O\left(n^{3}+m^{2}\right)$ clauses ensure that the underlying order is indeed linear, and that no two edges of the same page cross; for details refer to 3 .

For the dispersable case, we must additionally guarantee that no two edges with a common endvertex are assigned to the same page. This requirement, however, can be easily encoded by the following clauses:

$$
\neg \chi\left(e, e^{\prime}\right), \quad \forall e, e^{\prime} \text { with a common endvertex }
$$

Observe that there is no need to introduce new variables, and that the total number of constraints is not asymptotically affected. Using this adjustment, we proved that the dispersable book thickness of the Gray graph cannot be three, and that it admits a dispersable book embedding with four pages; see Fig. 18 in Appendix B. We summarize these findings in the following theorem. 
Theorem 2. The dispersable book thickness of the Gray graph is four.

Corollary 3. The Gray graph is not dispersable.

\section{3-connected 3-regular Bipartite Planar Graphs}

In the previous section, we demonstrated that the Gray graph, which is 3connected, 3-regular and bipartite, is not dispersable. This graph, however, is not planar, as it contains $K_{3,3}$ as minor. In the following, we prove that when adding planarity to the requirements, every such graph is dispersable. For the sake of simplicity, we refer to a 3-connected 3-regular bipartite planar graph as Barnette graph for short (due to the well-known Barnette's Conjecture [1] which states that every such graph is Hamiltonian).

Lemma 5. Let $G=(V, E)$ be an embedded Barnette graph and let $G^{*}=\left(V^{*}, E^{*}\right)$ be its dual. Then, there exists a 3-edge coloring $E_{r} \sqcup E_{g} \sqcup E_{b}=E$ for $G$, and a 3-vertex coloring $V_{r}^{*} \sqcup V_{g}^{*} \sqcup V_{b}^{*}=V^{*}$ for $G^{*}$ which satisfy the following three properties:

i. Every facial cycle of $G$ is bichromatic, i.e., the edges on a facial cycle of $G$ alternate between two colors.

ii. Every face of $G$ is colored differently from its bounding edges.

iii. The edges of $G^{*}$ that connect vertices of $V_{g}^{*}$ to vertices of $V_{b}^{*}$ are in one-toone correspondence with the edges of $E_{r}$, and induce a connected subgraph.

Proof. Since $G$ is 3-regular and bipartite, $G^{*}$ is maximal planar and every vertex has even degree. By the 3 -color theorem, $G^{*}$ is 3 -vertex colorable [27]. Also, since $G^{*}$ is maximal planar and its chromatic number is $3, G^{*}$ is uniquely 3colorable [8, i.e., it has a unique 3 -vertex coloring up to permutation of the colors. Let $V_{\mathrm{r}}^{*}, V_{\mathrm{g}}^{*}$ and $V_{\mathrm{b}}^{*}$ be this 3 -vertex coloring of $G^{*}$.

We first show Property (iii). Every edge $e$ of $G$ bounds two faces that are colored differently in $G^{*}$. Hence, we can assign to $e$ the third unused color. Since every vertex $v$ of $G$ is incident to three faces (which pairwise do not share the same color in $G^{*}$ ), no two edges incident to $v$ have the same color. Thus, the result is a proper 3-edge coloring $E_{\mathrm{r}}, E_{\mathrm{g}}, E_{\mathrm{b}}$ of $G$.

Now Property (i) follows easily: On every facial cycle $p$ of $G$, two edges sharing an endpoint have distinct colors. By Property (ii), their colors are different from the color of $p$ in $G^{*}$. Thus, every face of $G$ is bichromatic.

Next we show Property (iii). By Property (iii), any edge of $G^{*}$ that corresponds to an edge of $E_{\mathrm{r}}$ has one endpoint in $V_{\mathrm{g}}^{*}$ and one in $V_{\mathrm{b}}^{*}$. Conversely, by construction every edge of $G^{*}$ in the induced subgraph of $V_{\mathrm{g}}^{*} \cup V_{\mathrm{b}}^{*}$ corresponds to an edge in $E_{\mathrm{r}}$ of $G$. Hence, the edges of $G^{*}$ that connect vertices of $V_{\mathrm{g}}^{*}$ to vertices of $V_{\mathrm{b}}^{*}$ are indeed in one-to-one correspondence with the edges of $E_{\mathrm{r}}$. Property (iii) follows by a result of Chartrand and Geller [8], who showed that for any $k$-vertex coloring of a uniquely $k$-colorable graph, the subgraph induced by any two of the $k$ colors is connected. 
In the following, we show that it is always possible to determine a dispersable order for a Barnette graph $G$ such that the coloring of Lemma 5 for $G$ is a feasible page assignment. In particular, the green edges will always connect vertices that are consecutive in the dispersable order, which implies that they can be "merged" with either the red or the blue edges, yielding thus a (non-dispersable) two-page book embedding of $G$. Our construction is based on determining a so-called subhamiltonian cycle $C$ for $G$, that is, a cyclic ordering of the vertices such that when adding any missing edges between consecutive vertices the resulting graph remains planar (thus, $C$ becomes a Hamiltonian cycle). This subhamiltonian cycle partitions the edges of $G$, such that the green and the red edges are either inside or on $C$, while the blue edges are in the exterior of $C$. With these properties in mind, we now state the main result of this section.

Theorem 3. Let $G=(V, E)$ be an embedded Barnette graph. Then, there exists a 3-edge coloring $E_{r} \sqcup E_{g} \sqcup E_{b}=E$ of $G$ and a subhamiltonian cycle $C$ satisfying the following properties:

i. every $e \in E_{r}$ is in the interior of $C$ or on $C$,

ii. every $e \in E_{b}$ is in the exterior of $C$ or on $C$,

iii. every $e \in E_{g}$ is on $C$.

Proof. In the proof, we assume that $E_{\mathrm{r}} \sqcup E_{\mathrm{g}} \sqcup E_{\mathrm{b}}$ is a 3-edge coloring of $G$, and that $V_{\mathrm{r}}^{*} \sqcup V_{\mathrm{g}}^{*} \sqcup V_{\mathrm{b}}^{*}$ is a 3 -vertex coloring of the dual $G^{*}=\left(V^{*}, E^{*}\right)$ of $G$ satisfying the Properties infii of Lemma 5 By Lemma 5 iii the subgraph $G_{b g}^{*}$ of $G^{*}$ induced by $V_{\mathrm{g}}^{*} \cup V_{\mathrm{b}}^{*}$ is connected. Hence, we can construct a spanning tree $T^{*}$ of $G_{b g}^{*}$. This tree and the one-to-one correspondence between the edges of $E_{\mathrm{r}}$ and the edges of $G_{b g}^{*}$ yield a partition of $E_{\mathrm{r}}$ into two sets $T_{\mathrm{r}}$ and $N_{\mathrm{r}}$, such that $T_{\mathrm{r}} \sqcup N_{\mathrm{r}}=E_{\mathrm{r}}$, as follows. An edge $e \in E_{\mathrm{r}}$ belongs to $T_{\mathrm{r}}$, if the edge of $G^{*}$ corresponding to $e$ belongs to $\mathcal{T}^{*}$. Otherwise, $e$ belongs to $N_{\mathrm{r}}$. We also assume $\mathcal{T}^{*}$ to be rooted at a leaf $\rho$, such w.l.o.g. $\rho \in V_{\mathrm{b}}^{*}$.

The proof is given by a recursive geometric construction of the subhamiltonian cycle $C$. Consider an arbitrary edge $(u, v) \in T_{\mathrm{r}}$ of $G$, and let $p$ and $q$ be the faces to its left and the right side, respectively, as we move along $(u, v)$ from $u$ to $v$. Then, $(p, q)$ is an edge of $\mathcal{T}^{*}$. Since $\mathcal{T}^{*}$ is a tree, the removal of $(p, q)$ results in two trees $\mathcal{T}_{p}^{*}$ and $\mathcal{T}_{q}^{*}$. W.l.o.g. we assume that $\rho$ belongs to $\mathcal{T}_{p}^{*}$. For the recursive step of our algorithm, we assume that we have already computed a simple and plane cycle $C_{p}$ for the subgraph $G_{p}=\left(V_{p}, E_{p}\right)$ of $G$ induced by the vertices of the faces of $G$ in $\mathcal{T}_{p}^{*}$, which satisfies the following additional invariants:

I.1 edge $(u, v)$ is on $C_{p}$,

I.2 every edge $e \in T_{\mathrm{r}} \cap E_{p}$ is in the interior of $C_{p}$ or on $C_{p}$,

I.3 every edge $e \in E_{\mathrm{b}} \cap E_{p}$ is in the exterior of $C_{p}$ or on $C_{p}$,

I.4 every edge $e \in E_{\mathrm{g}} \cap E_{p}$ is on $C_{p}$, and

I.5 every edge $e \in N_{\mathrm{r}}$ that bounds two faces $h, h^{\prime}$, with $h \in \mathcal{T}_{p}^{*}$ and $h^{\prime} \notin \mathcal{T}_{p}^{*}$, is such that:

i. if $h \in V_{\mathrm{b}}^{*}$, then both endpoints of $e$ are on $C_{p}$,

ii. if $h \in V_{\mathrm{g}}^{*}$, then none of the endpoints of $e$ is on $C_{p}$. 


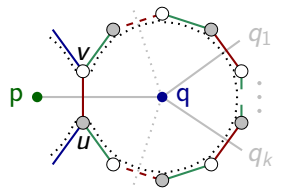

(a) $q \in V_{\mathrm{b}}^{*}$

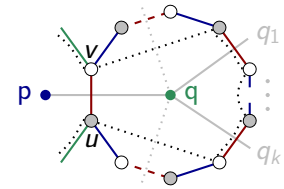

(b) $q \in V_{\mathrm{g}}^{*}$

Fig. 14: Illustration for Theorem 3. The solid (dotted) gray edges belong to $\mathcal{T}^{*}\left(G_{b g}^{*} \backslash\right.$ $\mathcal{T}^{*}$ ). The solid (dashed) red edges belong to $T_{\mathrm{r}}\left(N_{\mathrm{r}}\right)$. Cycle $C_{q}$ is drawn dotted black.

Let $G_{q}=\left(V_{q}, E_{q}\right)$ be the subgraph of $G$ induced by the vertices of the faces of $G$ in $\mathcal{T}_{q}^{*}$. Let also $q_{1}, \ldots, q_{k}$, with $k \geq 0$, be the children of $q$ in $\mathcal{T}^{*}$ (if any). We proceed by considering two cases; $q \in V_{\mathrm{b}}^{*}$ and $q \in V_{\mathrm{g}}^{*}$; see Figs. 14a and 14b. respectively. Note that by Lemma 5 i i and 5 lii in the former case, the edges of $q$ alternate between red and green, while in the latter case between red and blue.

Assume first that $q \in V_{\mathrm{b}}^{*}$. We remove from $C_{p}$ the edge $(u, v)$, which exists by Invariant I.1. This results in a path from $u$ to $v$. The cycle $C_{q}$ that is obtained by this path and the path from $u$ to $v$ in face $q$ is a cycle for $\mathcal{T}_{p}^{*} \cup\{q\}$, which satisfies Invariants I 1 I 5 as we discuss in the following.

Since $q$ is a face, it has no chords. Hence, the only chord we added to $C_{q}$ is $(u, v)$ which belongs to $T_{\mathrm{r}}$. This implies that Invariants I $2 \mathrm{I}, 4$ are satisfied. Since $q \in V_{\mathrm{b}}^{*}$, in order to guarantee Invariant 5i. we have to ensure that all edges of $q$ that belong to $N_{\mathrm{r}}$ belong to $C_{q}$. However, this trivially holds, since every edge of $q$ is on $C_{q}$ except for edge $(u, v)$, which however belongs to $T_{\mathrm{r}}$ (and thus not to $N_{\mathrm{r}}$ ). To guarantee Invariant I.1. we have to ensure that the edge of $G$ shared between $q$ and each child $q_{i}$ of $q$ in $\mathcal{T}^{*}$ is on $C_{q}(i=1, \ldots, k)$. Since the only edge of $q$ that is not on $C_{q}$ is the edge $(u, v)$, which is not shared by any child of $q$, it follows that Invariant I 1 is also maintained. It remains to also prove that $C_{q}$ is simple and plane. The latter property is straight-forward. For the former property, assume for a contradiction that cycle $C_{q}$ is not simple. Since $C_{p}$ is simple, one of the newly introduced vertices, call it $w$, is in $C_{p}$. Since $q \in V_{\mathrm{b}}^{*}, w$ is incident to a red edge, say $(w, z)$, in $q$. Let $q^{\prime} \in V_{\mathrm{g}}^{*}$ be the face of $G$ that is incident to $(w, z)$ and different from $q$. Since $w$ belongs to $C_{p}$, face $q^{\prime}$ belongs to $\mathcal{T}_{p}^{*}$. Hence, $(w, z) \in N_{\mathrm{r}}$. Since $q^{\prime} \in V_{\mathrm{g}}^{*}$, none of the endpoints of $(w, z)$ is on $C_{p}$ due to Invariant I 5ii a contradiction. Hence, $C_{q}$ is indeed simple.

Assume now that $q \in V_{\mathrm{g}}^{*}$. If $q$ is a leaf in $\mathcal{T}^{*}$ (i.e., the only edge incident to $q$ that belongs to $T_{\mathrm{r}}$ is edge $\left.(u, v)\right)$, then $C_{p}$ is a (simple and plane) cycle also for $\mathcal{T}_{p}^{*} \cup\{q\}$, which trivially satisfies Invariants I.1. I. 5 . So, we assume w.l.o.g. that $q$ is not a leaf in $\mathcal{T}^{*}$. It follows that there exist edges of $q$, different from $(u, v)$, that belong to $T_{\mathrm{r}}$. Denote by $w_{1}, \ldots, w_{\ell}$ the endvertices of these edges as they appear in a clockwise traversal of $q$ starting from $u$. We remove from $C_{p}$ the edge $(u, v)$, which exists by Invariant I 1 . This results in a path from $u$ to $v$. The cycle $C_{q}$ that is obtained by this path and the path $u \rightarrow w_{1} \rightarrow \ldots \rightarrow w_{\ell} \rightarrow v$ is a cycle for $\mathcal{T}_{p}^{*} \cup\{q\}$, which satisfies Invariants I, 1 I 5 as we prove in the following. 


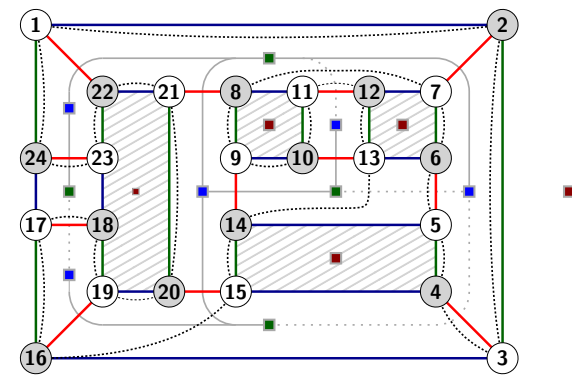

(a)

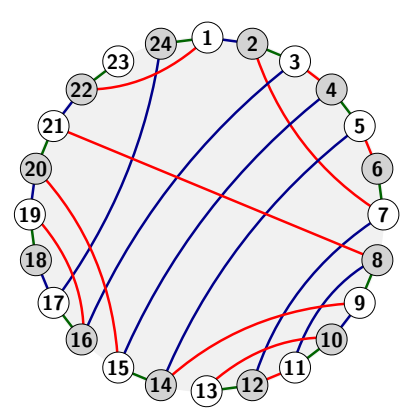

(b)

Fig. 15: Illustration of (a) a sample subhamiltonian cycle (dotted drawn), and (b) a corresponding dispersable book embedding with three pages produced by our algorithm.

Since $C_{q}$ passes through all edges of $q$ that belong to $T_{\mathrm{r}}$ and since these edges are the only edges that are shared between $q$ and the children of $q$ in $\mathcal{T}^{*}$, Invariant I 1 is satisfied. Invariants I 2 is satisfied, because edge $(u, v)$, which belongs to $T_{\mathrm{r}}$, is an internal chord of $C_{q}$, and all edges of $q$ that belong to $T_{\mathrm{r}}$ are on $C_{q}$. For Invariant I 3 , we argue as follows. Consider an edge $e$ of $q$ that belongs to $E_{\mathrm{b}}$. If both the (red) edges that precede and that follow $e$ in $q$ belong to $T_{\mathrm{r}}$, then $e$ is on $C_{q}$; otherwise, $e$ is not in the interior of $C_{q}$. Hence, Invariant I 3 is satisfied. Invariant I 4 is trivially satisfied, as $q$ belongs to $V_{\mathrm{g}}^{*}$ and therefore by Lemma 5 iii does not contain any edge of $E_{\mathrm{g}}$. Finally, Invariant I 5 ii is satisfied, since the only vertices of $q$ that belong to $C_{q}$ are the endvertices of the edges of $q$ that belong to $T_{\mathrm{r}}$ (and thus not to $N_{\mathrm{r}}$ ). We conclude this case by mentioning that $C_{q}$ can be easily proven to be plane and that the fact that $C_{q}$ is simple can be proved symmetrically to the case in which $q$ belongs to $V_{\mathrm{b}}^{*}$.

The base of our recursive algorithm corresponds to the face $\rho \in V_{\mathrm{b}}^{*}$ that is the root of $\mathcal{T}^{*}$. In this case, by setting $C_{\rho}$ to be the facial cycle $\rho$, we trivially satisfy all invariant properties of our algorithm; see Fig. 15 for an example.

Once we traverse $\mathcal{T}^{*}$, we have computed a simple and plane cycle $C$, which by Invariants 2, 4, satisfies Properties in iii of our theorem. We show that $C$ is a subhamiltonian cycle of $G$ as follows. Since $\mathcal{T}^{*}$ is a spanning tree of $G_{b g}^{*}$, every green edge of $G$ bounds a face that is in $\mathcal{T}^{*}$, and by Invariant 4 we may assume that both its endpoints are consecutive along $C$. As every vertex is incident to a green edge, it follows that $C$ is indeed a subhamiltonian cycle of $G$.

Theorem 3 implies that every Barnette graph admits a proper 3-edge coloring and a two-page book embedding, in which the green edges connect consecutive vertices along the spine, while the red and blue edges must be on different pages. To achieve our initial goal, which was to show that every Barnette graph is dispersable, we only have to assign the green edges to an additional third page. This result is summarized in the following corollary.

Corollary 4. Every Barnette graph is dispersable. 
In relation to Barnette's conjecture, our result does not guarantee the existence of a Hamiltonian cycle. However, it guarantees the existence of a subhamiltonian cycles, which contains roughly $\frac{2 n}{3}$ edges, where $n$ is the number of vertices of the graph. More precisely, all green edges, which are $\frac{n}{2}$ in total, are part of this cycle. Moreover, the red edges that belong to the set $N_{\mathrm{r}}$ in the proof of Theorem 3 are by construction also part of this cycle. As a 3-regular planar graph has exactly $\frac{n}{2}+2$ faces, we may assume w.l.o.g. that the number of nodes in $G_{b g}^{*}$ is at most

$$
\frac{2}{3}\left(\frac{n}{2}+2\right)=\frac{1}{3}(n+4),
$$

which implies that

$$
\left|T_{\mathrm{r}}\right| \leq \frac{1}{3}(n+4)-1 .
$$

As a result, the number of edges in $N_{\mathrm{r}}$ is at least

$$
\frac{n}{2}-\left(\frac{1}{3}(n+4)-1\right)=\frac{n}{6}-\frac{1}{3} .
$$

So, in total the number of green edges and the number of red edges in $N_{\mathrm{r}}$ is at least $\frac{n}{2}+\frac{n}{6}-\frac{1}{3}=\frac{2 n}{3}-\frac{1}{3}$, as initially claimed.

\section{Conclusions}

In this paper, we studied dispersable book embeddings, and we demonstrated that for $k=3,4$, the dispersable book thickness of a $k$-regular bipartite graph is not necessarily $k$, thus disproving an old conjecture by Bernhart and Kainen 4]. There exists a number of interesting related questions raised by our work.

1. A natural question to ask is whether there exist a non-dispersable bipartite graph for every $k \geq 5$. Our computational experiments confirm the hypothesis for $k=5,6$. For larger values of $k$, combinatorial proofs are needed.

2. A common property of the Folkman graph and of the Gray graph is that both are not vertex-transitive. So, it is natural to ask whether vertex-transitive regular bipartite graphs are dispersable.

3. We conjecture that all (i.e., not necessarily 3-connected) 3-regular bipartite planar graphs are dispersable; proving or disproving the conjecture is a possible future direction.

4. Is it possible to guarantee an upper bound on the dispersable book thickness of $k$-regular bipartite graphs (e.g., $k+1$ )?

5. What is the complexity of the testing problem whether a given graph is dispersable? The question is of interest also in the case of fixed linear order or fixed edge-to-page assignment.

6. More generally, it would be interesting to study the dispersable book thickness of (non-regular) bipartite planar graphs of maximum degree $\Delta(G)$. Extensive computational experiments indicate that all such graphs admit dispersable book embeddings with $\Delta(G)$ pages. 


\section{References}

1. Barnette, D.W.: Conjecture 5. In: Tutte, W.T. (ed.) Recent progress in combinatorics. pp. xiv +347 . Proceedings of the Third Waterloo Conference on Combinatorics, Academic Press, New York-London (1969)

2. Bekos, M.A., Gronemann, M., Raftopoulou, C.N.: Two-page book embeddings of 4-planar graphs. Algorithmica 75(1), 158-185 (2016)

3. Bekos, M.A., Kaufmann, M., Zielke, C.: The book embedding problem from a satsolving perspective. In: Giacomo, E.D., Lubiw, A. (eds.) Graph Drawing. LNCS, vol. 9411, pp. 125-138. Springer (2015)

4. Bernhart, F., Kainen, P.C.: The book thickness of a graph. Journal of Combinatorial Theory, Series B 27(3), 320-331 (1979)

5. Blankenship, R.: Book Embeddings of Graphs. Ph.D. thesis, Louisiana State University (2003)

6. Bouwer, I.: On edge but not vertex transitive regular graphs. Journal of Combinatorial Theory, Series B 12(1), 32 - 40 (1972)

7. Buss, J.F., Shor, P.W.: On the pagenumber of planar graphs. In: DeMillo, R.A. (ed.) ACM Symposium on Theory of Computing. pp. 98-100. ACM (1984)

8. Chartrand, G., Geller, D.P.: On uniquely colorable planar graphs. Journal of Combinatorial Theory 6(3), 271-278 (1969)

9. Chung, F.R.K., Leighton, F.T., Rosenberg, A.L.: Embedding graphs in books: A layout problem with applications to VLSI design. SIAM. Journal on Algebraic and Discrete Methods 8(1), 33-58 (1987)

10. Cornuéjols, G., Naddef, D., Pulleyblank, W.: Halin graphs and the travelling salesman problem. Mathematical Programming 26(3), 287-294 (1983)

11. Dujmovic, V., Wood, D.: Graph treewidth and geometric thickness parameters. Discrete Computational Geomentry 37(4), 641-670 (2007)

12. Dujmovic, V., Wood, D.R.: On linear layouts of graphs. Discrete Mathematics \& Theoretical Computer Science 6(2), 339-358 (2004)

13. Folkman, J.: Regular line-symmetric graphs. Journal of Combinatorial Theory 3(3), $215-232(1967)$

14. de Fraysseix, H., de Mendez, P.O., Pach, J.: A left-first search algorithm for planar graphs. Discrete \& Computational Geometry 13, 459-468 (1995)

15. Heath, L.S.: Embedding planar graphs in seven pages. In: FOCS. pp. 74-83. IEEE Computer Society (1984)

16. Heath, L.S.: Algorithms for Embedding Graphs in Books. Ph.D. thesis, University of N. Carolina (1985)

17. Heath, L.S., Leighton, F.T., Rosenberg, A.L.: Comparing queues and stacks as machines for laying out graphs. SIAM Journal of Discrete Mathematics 3(5), 398412 (1992)

18. Hoske, D.: Book embedding with fixed page assignments. Bachelor thesis, Karlsruhe Institute for Technology (2012)

19. Kainen, P.C.: Crossing-free matchings in regular outerplane drawings. In: Knots in Washington XXIX. George Washington University, Washington, DC, USA (2009), http://faculty.georgetown.edu/kainen/circLayouts.pdf

20. Kainen, P.C., Overbay, S.: Extension of a theorem of Whitney. Applied Mathematics Letters 20(7), 835-837 (2007)

21. Malitz, S.: Genus $g$ graphs have pagenumber $O(\sqrt{q})$. Journal of Algorithms $17(1)$, 85-109 (1994) 
22. Malitz, S.: Graphs with E edges have pagenumber $O(\sqrt{E})$. Journal of Algorithms 17(1), 71-84 (1994)

23. Nishizeki, T., Chiba, N.: Planar Graphs: Theory and Algorithms, chap. 10. Hamiltonian Cycles, pp. 171-184. Dover Books on Mathematics, Courier Dover Publications (2008)

24. Ollmann, T.: On the book thicknesses of various graphs. In: Hoffman, F., Levow, R., Thomas, R. (eds.) Southeastern Conference on Combinatorics, Graph Theory and Computing. Congressus Numerantium, vol. VIII, p. 459 (1973)

25. Overbay, S.B.: Generalized book embeddings. Ph.D. thesis, Colorado State University (1998)

26. Rosenberg, A.L.: The Diogenes approach to testable fault-tolerant arrays of processors. IEEE Transactions on Computers C-32(10), 902-910 (1983)

27. Steinberg, R.: The state of the three color problem. In: Gimbel, J., Kennedy, J.W., Quintas, L.V. (eds.) Quo Vadis, Graph Theory?, Annals of Discrete Mathematics, vol. 55, pp. 211-248. Elsevier (1993)

28. Tarjan, R.: Sorting using networks of queues and stacks. Journal of the ACM 19(2), 341-346 (1972)

29. Wigderson, A.: The complexity of the Hamiltonian circuit problem for maximal planar graphs. Tech. Rep. TR-298, EECS Department, Princeton University (1982)

30. Yannakakis, M.: Embedding planar graphs in four pages. Journal of Computer and System Sciences 38(1), 36-67 (1989)

31. Gerbracht, E.: Eleven unit distance embeddings of the Heawood graph. CoRR abs/0912.5395 (2009) 


\section{Appendix}

\section{A Book Embeddings of the Folkman Graph}

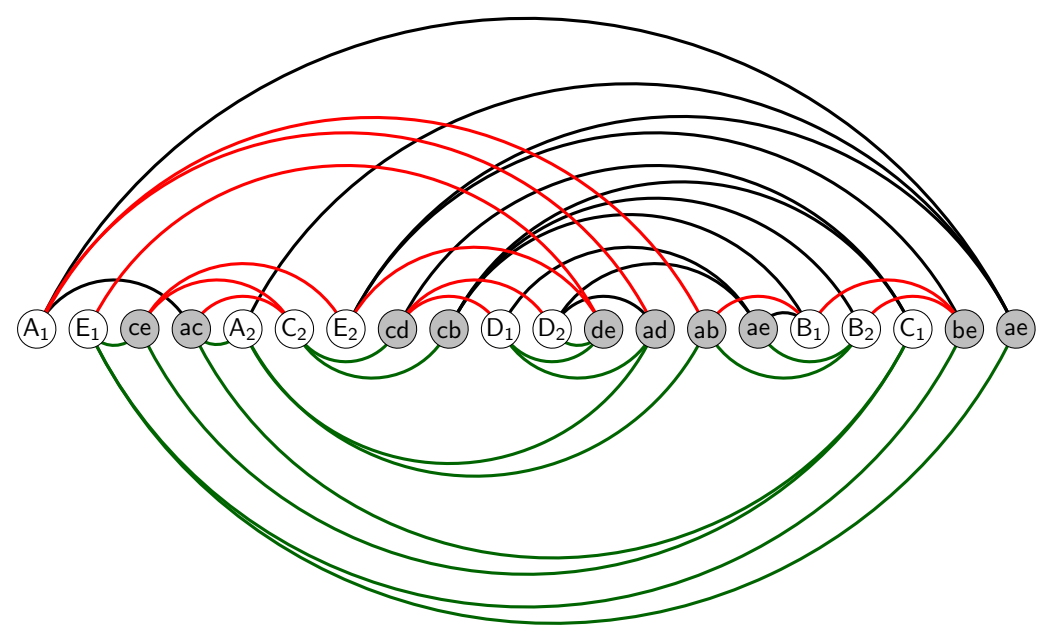

(a)

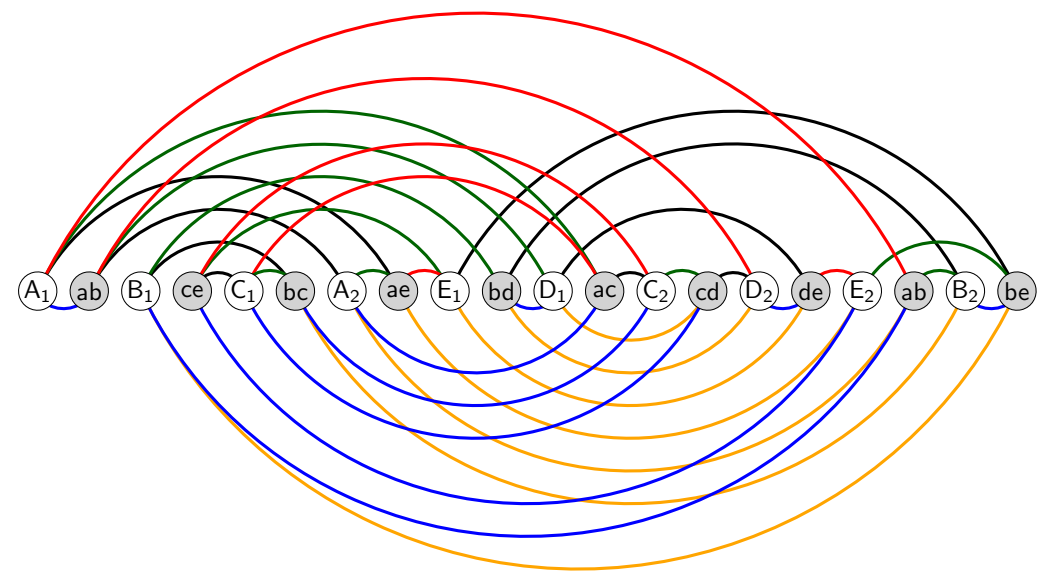

(b)

Fig. 16: Different book embeddings of the Folkman graph: (a) ordinary with 3 pages, and (b) dispersable with 5 pages. 


\section{B Book Embeddings of the Gray Graph}

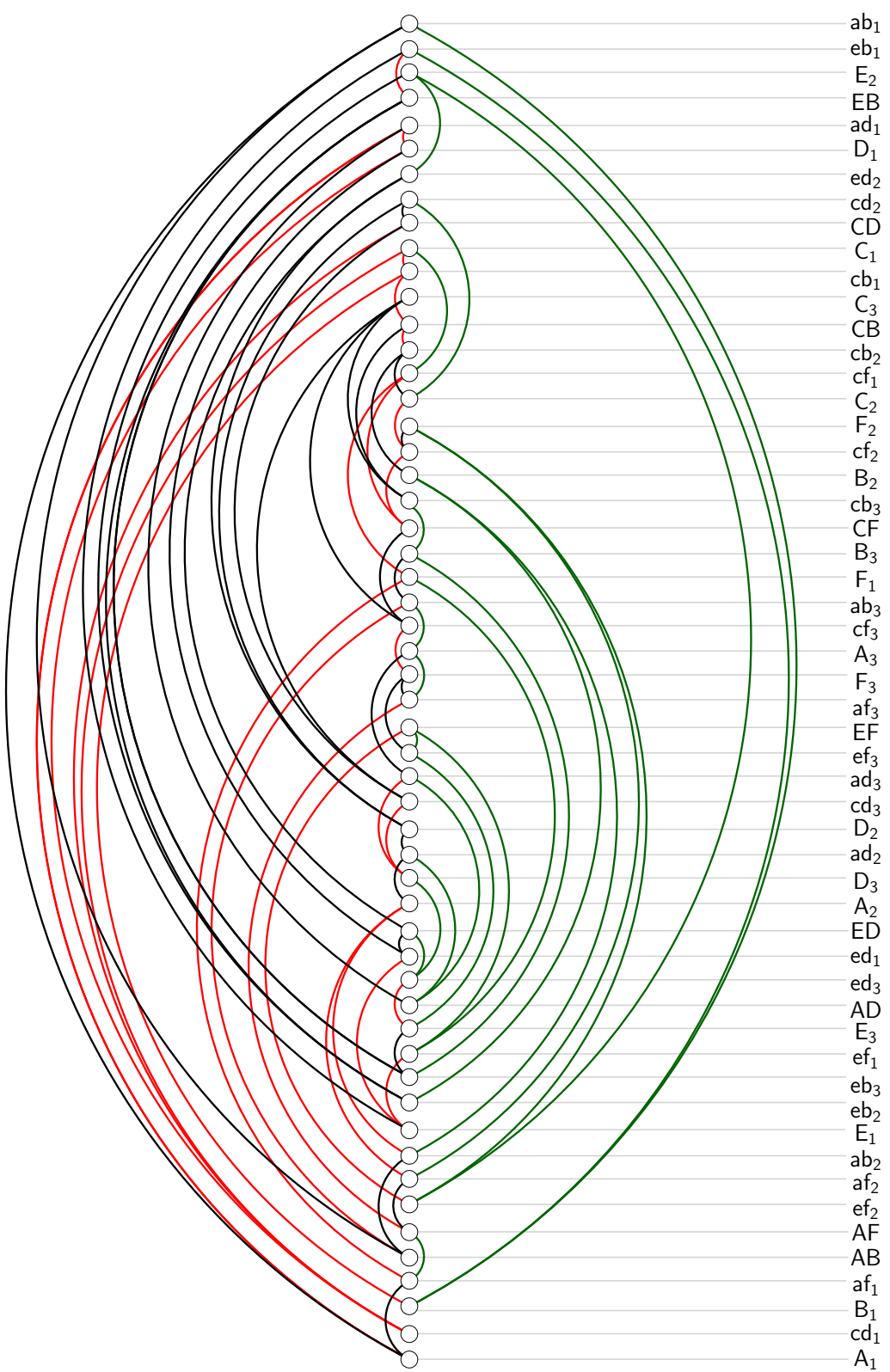

Fig. 17: An ordinary book embedding of the Gray graph with 3 pages. 


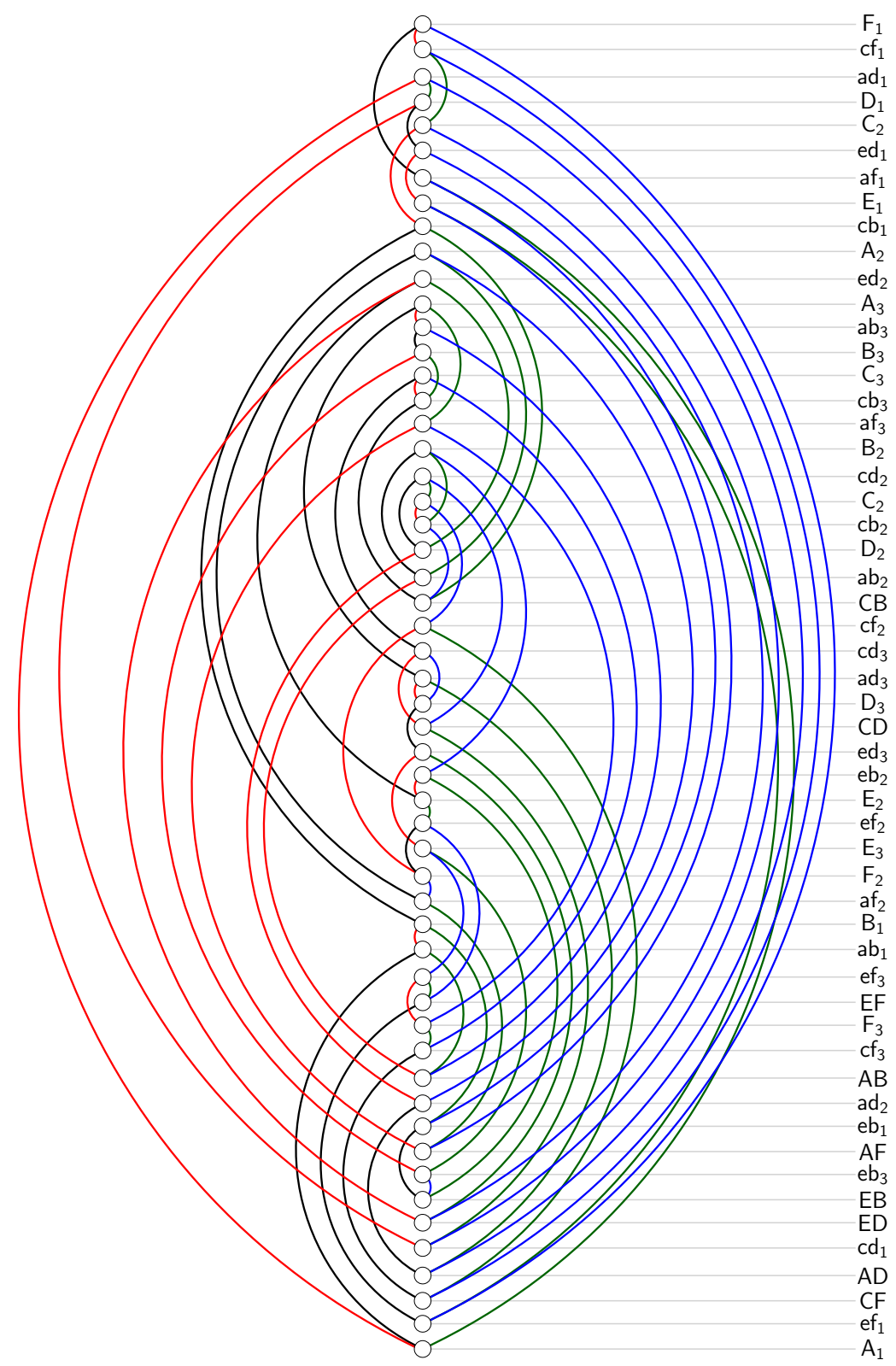

Fig. 18: A dispersable book embedding of the Gray graph with 4 pages. 